\title{
Neutral oxygen spectral line formation revisited with new collisional data: large departures from LTE at low metallicity
}

\author{
D. Fabbian ${ }^{1, \star}$, M. Asplund ${ }^{2}$, P. S. Barklem ${ }^{3}$, M. Carlsson ${ }^{4}$, and D. Kiselman ${ }^{5}$
}

\author{
1 Research School of Astronomy \& Astrophysics, The Australian National University, Mount Stromlo Observatory, Cotter Road, \\ Weston ACT 2611, Australia \\ e-mail: damian@iac.es \\ 2 Max Planck Institute for Astrophysics, Postfach 1317, 85741 Garching b. München, Germany \\ 3 Department of Physics and Astronomy, Uppsala University, Box 515, 751-20 Uppsala, Sweden \\ 4 Institute of Theoretical Astrophysics, University of Oslo, PO Box 1029, Blindern, 0315 Oslo, Norway \\ 5 The Institute for Solar Physics of the Royal Swedish Academy of Sciences, AlbaNova University Centre, 10691 Stockholm, \\ Sweden
}

Received 24 February 2008 / Accepted 17 February 2009

\section{ABSTRACT}

Aims. A detailed study is presented, including estimates of the impact on elemental abundance analysis, of the non-local thermodynamic equilibrium (non-LTE) formation of the high-excitation neutral oxygen $777 \mathrm{~nm}$ triplet in model atmospheres representative of stars with spectral types F to K.

Methods. We have applied the statistical equilibrium code MULTI to a number of plane-parallel MARCS atmospheric models covering late-type stars $\left(4500 \leq T_{\text {eff }} \leq 6500 \mathrm{~K}, 2 \leq \log g \leq 5[\mathrm{cgs}]\right.$, and $\left.-3.5 \leq[\mathrm{Fe} / \mathrm{H}] \leq 0\right)$. The atomic model employed includes, in particular, recent quantum-mechanical electron collision data.

Results. We confirm that the O I triplet lines form under non-LTE conditions in late-type stars, suffering negative abundance corrections with respect to LTE. At solar metallicity, the non-LTE effect, mainly attributed in previous studies to photon losses in the triplet itself, is also driven by an additional significant contribution from line opacity. At low metallicity, the very pronounced departures from LTE are due to overpopulation of the lower level $\left(3 \mathrm{~s}^{5} \mathrm{~S}^{\mathrm{o}}\right)$ of the transition. Large line opacity stems from triplet-quintet intersystem electron collisions, a form of coupling previously not considered or seriously underestimated. The non-LTE effects generally become severe for models (both giants and dwarfs) with higher $T_{\text {eff }}$. Interestingly, in metal-poor turn-off stars, the negative non-LTE abundance corrections tend to rapidly become more severe towards lower metallicity. When neglecting $\mathrm{H}$ collisions, they amount to as much as $\left|\Delta \log \epsilon_{\mathrm{O}}\right| \sim 0.9$ dex and $\sim 1.2$ dex, respectively at $[\mathrm{Fe} / \mathrm{H}]=-3$ and $[\mathrm{Fe} / \mathrm{H}]=-3.5$. Even when such collisions are included, the LTE abundance remains a serious overestimate, correspondingly by $\left|\Delta \log \epsilon_{\mathrm{O}}\right| \sim 0.5 \mathrm{dex}$ and $\sim 0.9 \mathrm{dex}$ at such low metallicities. Although the poorly known inelastic hydrogen collisions thus remain an important uncertainty, the large metallicity-dependent non-LTE effects seem to point to a resulting "low" (compared to LTE) $[\mathrm{O} / \mathrm{Fe}]$ in metal-poor halo stars.

Conclusions. Our results may be important in solving the long-standing $[\mathrm{O} / \mathrm{Fe}]$ debate. When applying the derived non-LTE corrections, the LTE oxygen abundance inferred from the $777 \mathrm{~nm}$ permitted triplet will be decreased substantially at low metallicity. If the classical Drawin formula is employed for $\mathrm{O}+\mathrm{H}$ collisions, the derived $[\mathrm{O} / \mathrm{Fe}]$ trend becomes almost flat below $[\mathrm{Fe} / \mathrm{H}] \sim-1$, in better agreement with recent literature estimates generally obtained from other oxygen abundance indicators. A value of $[\mathrm{O} / \mathrm{Fe}] \lessgtr+0.5$ may therefore be appropriate, as suggested by standard theoretical models of type II supernovae nucleosynthetic yields. If neglecting impacts with $\mathrm{H}$ atoms instead, $[\mathrm{O} / \mathrm{Fe}]$ decreases towards lower $[\mathrm{Fe} / \mathrm{H}]$, which would open new questions. Our tests using ATLAS model atmospheres show that, though non-LTE corrections for metal-poor dwarfs are smaller (by $\sim 0.2$ dex when adopting efficient $\mathrm{H}$ collisions) than in the MARCS case, our main conclusions are preserved, and that the LTE approach tends to seriously overestimate the $\mathrm{O}$ abundance at low metallicity. However, in order to finally reach consistency between oxygen abundances from the different available spectral features, it is of high priority to reduce the large uncertainty regarding $\mathrm{H}$ collisions, to undertake a full investigation of the interplay of non-LTE and 3D effects, and to clarify the issue of the temperature scale at low metallicity.

Key words. line: formation - stars: abundances - stars: late-type - Galaxy: evolution

\section{Introduction}

After the primordially ubiquitous $\mathrm{H}$ and $\mathrm{He}$, oxygen is the third most abundant chemical element in the Universe, and the first among those produced via stellar nucleosynthesis. It is a catalyst in the CNO process, which is the main conversion channel of $\mathrm{H}$ into $\mathrm{He}$ in the interiors of stars more massive than the Sun. The oxygen content of stars and its abundance ratio with other chemical elements derived from observations of metal-poor halo

\footnotetext{
* Current address: Instituto de Astrofísica de Canarias, Calle Via Láctea s/n, E38205, La Laguna, Tenerife, España.
}

stars are crucial (e.g. Matteucci \& François 1992) to constraining galactic chemical evolution (GCE) models. Oxygen, like the other $\alpha$-elements, has been known for a long time (e.g. Conti et al. 1967) to show an overabundance with respect to iron in stars of low metallicity. This is expected since its main production site is in massive stars which end their lives as Type II supernovae (SNe II, Arnett 1978; Clayton 2003). Due to the longer lifetimes of their precursors, the delayed release of additional iron from type Ia $\mathrm{SNe}$ starts to affect $[\mathrm{O} / \mathrm{Fe}]^{1}$ only at a later

\footnotetext{
${ }^{1}$ By definition, $[\mathrm{A} / \mathrm{X}]=\log \left(N_{\mathrm{A}} / N_{\mathrm{X}}\right)_{*}-\log \left(N_{\mathrm{A}} / N_{\mathrm{X}}\right)_{\odot}$.
} 
stage. The oxygen abundance in unevolved very metal-poor stars should therefore reflect the enrichment by the very first generations of SNe.

The oxygen abundance at low metallicity has been the focus of a plethora of studies. Still, a long-standing debate exists about the exact $[\mathrm{O} / \mathrm{Fe}]$ trend in metal-poor stars (see e.g. discussion in Asplund 2005; and Meléndez et al. 2006, and references therein). Here, we only briefly mention some pertinent works related to the subject.

Neutral oxygen lines used in abundance studies of solar-type stars include the high excitation line at $615.8 \mathrm{~nm}$, the weak forbidden [O I] lines at $630.0 \mathrm{~nm}$ and $636.4 \mathrm{~nm}$, and the infrared triplet at $777 \mathrm{~nm}$. The latter is the only strong oxygen atomic feature observable in spectra of late-type stars. While it is known to suffer from large non-LTE effects in early-type stars (Johnson et al. 1974; Baschek et al. 1977), there is little consensus on the estimated size of the abundance corrections for cooler stars of solar type. For example, the formation of the IR triplet lines in the Sun has been the object of a number of investigations. With a semiempirical approach, Altrock (1968) found that the non-LTE source function drops below the Planck function in the solar photosphere, a result later confirmed by the multilevel non-LTE study by Sedlmayr (1974). More recently, the non-LTE abundance corrections in the Sun have been estimated to be between -0.1 and -0.25 dex (Kiselman 1991, 1993; Gratton et al. 1999; Takeda 2003; Takeda \& Honda 2005; Asplund et al. 2004), mostly depending on whether efficient collisions with neutral $\mathrm{H}$ atoms are adopted or not. These authors explain the non-LTE line strengthening as due to photon losses in the lines, while the population (and thus the line opacity) of the lower level in the $777 \mathrm{~nm}$ transition is thought to stay close to LTE.

Non-LTE effects on oxygen lines in solar-type stars have been reviewed by Kiselman (2001). Statistical equilibrium calculations using a 16-level oxygen model atom (Kiselman 1991) have predicted an enhancement towards low metallicity of the (negative) non-LTE abundance corrections affecting the permitted $777 \mathrm{~nm}$ O I lines, suggesting that an LTE approach will seriously overestimate the derived oxygen abundance. The $[\mathrm{O} / \mathrm{Fe}]$ ratio derived in LTE using this abundance indicator would thus be increasingly reduced in metal-poor stars once non-LTE effects are considered. Other calculations have instead found almost $[\mathrm{Fe} / \mathrm{H}]$-independent non-LTE corrections (Takeda 2003).

Very high overabundances of oxygen compared to iron have occasionally been derived at low metallicity. Analysing the O I $777 \mathrm{~nm}$ lines in metal-poor dwarfs, Abia \& Rebolo (1989) found a steep monotonic increase in the oxygen-to-iron ratio for lower metallicities, reaching $[\mathrm{O} / \mathrm{Fe}]>1$ below $[\mathrm{Fe} / \mathrm{H}] \sim-2)$. They investigated non-LTE effects for the triplet lines but claimed that they should be less severe than -0.2 dex, therefore still leaving a residual increasing trend. Their high abundance estimates were criticized in King (1993) as mainly due to too low effective temperature estimates. Other authors (Gratton \& Ortolani 1986; Barbuy 1988; Sneden et al. 1991; Spite \& Spite 1991; Kraft et al. 1992; Carretta et al. 2000; Cayrel et al. 2004; Spite et al. 2005) found $[\mathrm{O} / \mathrm{Fe}] \simeq 0.4-0.6$ in late-type stars with $[\mathrm{Fe} / \mathrm{H}] \lesssim-1$, in particular using the $[\mathrm{OI}]$ lines in metal-poor giants. This nearly plateau-like behaviour (with oxygen content varying much more slowly than iron at low $[\mathrm{Fe} / \mathrm{H}]$, or even remaining constant) could be interpreted as the result of the contribution by type II SNe alone to early galactic nucleosynthesis (see e.g. Matteucci \& Greggio 1986). While some authors have reported a fair agreement between the near-IR $777 \mathrm{~nm}$ oxygen lines and the forbidden line (Mishenina et al. 2000; Nissen et al. 2002), others (Takeda 2003; Fulbright \& Johnson 2003) derived higher (up to several tenths of a dex) abundance values from the triplet. In particular - even when applying the non-LTE corrections derived in his own investigation to the observations Takeda still derived apparently too high (by several tenths of a dex) oxygen abundance from the IR triplet lines in metal-poor halo stars, a possible signal of underestimated non-LTE effects. At $[\mathrm{Fe} / \mathrm{H}] \lessgtr-3.5$, even more extreme examples of the oxygen conflict have been found, with differences of up to 1.55 dex between [O I]-based and triplet abundances, and exceptionally high $[\mathrm{O} / \mathrm{Fe}] \gtrsim 2$ oxygen enhancements (e.g. Depagne et al. 2002; Israelian et al. 2004).

Unfortunately, the neutral atomic oxygen lines, in particular the LTE-obeying [O I] line, tend to become vanishingly weak in metal-poor solar-type stars. Thus, molecular bands of $\mathrm{OH}$ in the UV (electronic transitions) and near IR (vibration-rotation lines) have also been used. The former are relatively strong and thus detectable in FGK stars down to low $[\mathrm{Fe} / \mathrm{H}]$, the latter instead require high $\mathrm{S} / \mathrm{N}$ and are only seen in spectra of cool stars $\left(T_{\text {eff }}<5000 \mathrm{~K}\right)$. Results from both are however affected by likely important 3D effects, in particular in the case of the UV lines (Asplund \& Garcia Perez 2001), which may also suffer from the problem of missing opacity (Balachandran \& Bell 1998; Asplund 2004) and possible issues related to continuum location, blends in the spectra, atmospheric extinction/cutoff and low CCD sensitivity. Various other uncertainties - e.g. on oscillator strengths for both molecular bands - and incomplete knowledge of non-LTE effects on molecular line formation may also be important. Generally higher values of oxygen abundance (compared to those from the weak IR molecular features) are derived from the $\mathrm{OH}$ lines in the UV (Balachandran et al. 2001; Meléndez \& Barbuy 2002; Barbuy et al. 2003), but the above effects may indeed already for the most part explain the large discrepancy. Other authors (Israelian et al. 1998; Boesgaard et al. 1999) have instead found reasonable agreement between the $O$ abundances derived for a sample of mainly turn-off stars from the two indicators. It seems that consistent oxygen abundances using all the different diagnostics, namely permitted, as well as forbidden and molecular lines, can hardly be obtained at low metallicity. Departures from homogeneity and from LTE have been invoked at various times to solve the inconsistency. A combination of other factors, including determination of stellar parameters/temperature scale, quality of observations and of reduced data, adopted solar abundances and so on may additionally be of concern and it is hard to disentagle the different effects. To complicate the issue further, one needs to consider that some authors have derived oxygen abundances at low metallicity from unevolved stars, others from giants, and it could be hypothesized that the lower oxygen values usually found for the latter are due to a depletion process in their atmospheres. However, Bessell et al. (1991) discussed evidence that oxygen abundances from $\mathrm{OH}$ were similar in dwarfs and giants. More recently, García Pérez et al. (2006) confirmed a reasonable agreement between $\mathrm{OH}$ and $[\mathrm{OI}]$ abundances in metal-poor subgiants, finding that O I triplet abundances instead give higher values even when corrected for non-LTE effects. It is therefore important to check if such residual discrepancy is linked to problems in the previous modeling of the formation of the permitted lines.

Thus, here we focus on investigating departures from LTE in 1D. Crucially, we explore the very low metallicity range, to understand if the non-LTE abundance corrections depend strongly on metallicity. In the next section, we describe our non-LTE calculations, and give results in Sect. 3. We then embark on a comparison with other non-LTE studies of the O I $777 \mathrm{~nm}$ absorption lines (Sect. 4). Finally, the last two sections are dedicated to a 


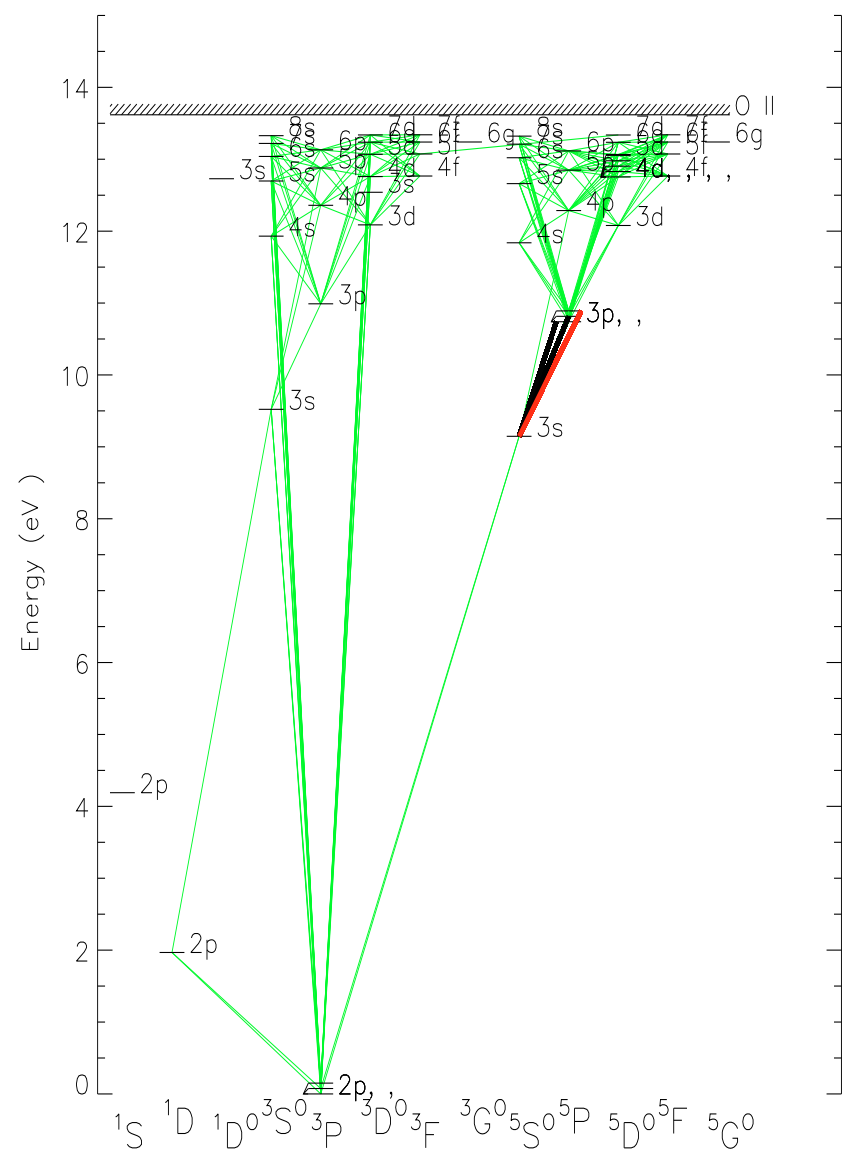

Fig. 1. Grotrian diagram of the atomic model we employed, showing the 53 energy levels of neutral oxygen and the single first ionization level. Lines connecting the various levels in the figure represent the 208 radiative bound-bound transitions included in the model. The $777 \mathrm{~nm}$ transitions are marked with thicker lines.

discussion of the impact on the $[\mathrm{O} / \mathrm{Fe}]$ ratio at low metallicity, and to some concluding remarks.

\section{Non-LTE calculations}

\subsection{Method}

The method employed is similar to that described in Fabbian et al. (2006). In order to derive the non-LTE populations of the $\mathrm{O}$ atomic levels and the strength of relevant spectral lines, it is necessary to solve the coupled rate and radiative transfer equations simultaneously. The code MULTI (Carlsson 1986) in its version 2.3 was used to perform the statistical equilibrium calculations for a number of MARCS model atmospheres (Gustafsson et al. 1975; Asplund et al. 1997), under the assumption that oxygen is a trace element and its departures from LTE will not affect the atmospheric structure. The atmospheric models tested have a microturbulence of $\xi=1$. A 54-level oxygen model atom (described below) was employed. We assumed complete redistribution in a Voigt profile for the formation of the lines.

\subsection{Atomic model}

We have constructed a model atom containing 54 energy levels and a total of 258 radiative transitions (208 b-b and $50 \mathrm{~b}-\mathrm{f}$ ). The Grotrian diagram of our atomic model is shown in Fig. 1. The necessary data for atomic level energies and corresponding statistical weights were taken from the NIST Atomic Spectra
Database $^{2}$ version 3. Neutral levels are included up to an excitation potential of $13.34 \mathrm{eV}$ (i.e. $\sim 0.28 \mathrm{eV}$ below the continuum). The model is complete to a principal quantum number of $n=7$. Fine-splitting of energy levels was taken into account where appropriate, in particular for the ground state and for the $3 p{ }^{5} \mathrm{P}$ level (upper level of the $777 \mathrm{~nm}$ O I triplet). For the transitions of importance in solar work which were used in Asplund et al. (2004), $f$-values are also from NIST while radiative and Stark parameters come from VALD 3 (Vienna Atomic Line Database, Piskunov et al. 1995, and successive updates). Data for boundfree photoionization are from the Opacity Project ${ }^{4}$ (Seaton 1987, and later updates). For collisional broadening due to $\mathrm{H}$ atoms, we adopt the classical Unsöld approximation. Our tests reveal that choosing an enhancement factor of two for the related damping constant does not affect our results significantly. The contribution to background opacity in the UV from lines of other elements was accounted for by compiling NIST and VALD values. In general, the data in the model atoms ${ }^{5}$ of Carlsson \& Judge (1993, hereafter CJ93) and Kiselman (1993, hereafter K93) were used (see those papers for description of the data sources) unless otherwise stated.

We have also included the most recent data for electronimpact excitation, available up to level $2 \mathrm{p}^{3} 4 \mathrm{f}^{3} \mathrm{~F}$ from quantummechanical calculations (Barklem 2007a), a novel feature of our model atom which has proven one of the essential improvements with respect to other studies. In particular, this has allowed us to include accurate rates for radiatively forbidden transitions due to electron collisions.

Since the estimated cross-sections were derived using LScoupling, they are null only for singlet-quintet transitions (total electronic spin conservation rule) and for collisionally forbidden $\mathrm{S}^{\mathrm{e}}-\mathrm{S}^{\mathrm{o}}$ transitions. In broad terms, the collisional cross-sections compare fairly well with available empirical data (within the experimental error bars, see Fig. 2 in Barklem 2007a). Although admittedly still subject to uncertainties due to e.g. pseudoresonances at higher energies, they should be more realistic than previous estimates. They are in general comparatively larger than $\mathrm{H}$ collisions by a few orders of magnitude, except for fine-splitting levels ${ }^{6}$. We stress here that the cross-sections for electron-impact collisions in the radiatively forbidden transition $3 \mathrm{~s}^{3} \mathrm{~S}^{\mathrm{o}}-3 \mathrm{~s}{ }^{5} \mathrm{~S}^{\mathrm{o}}$ are among the largest in the calculations of Barklem (2007a). In particular (see Fig. 1 in that work) they are larger than the other values for transitions arising from the lowest levels (and between the first seven energy levels in our atomic model).

Due to the deficiency of metals, and thus the decreased amount of free electrons in their atmosphere, rates due to collisions with neutral $\mathrm{H}$ atoms may be particularly important in low-metallicity stars. The approximation we adopted for excitation and ionization via inelastic collisions with $\mathrm{HI}$ is based on recipes by Steenbock \& Holweger (1984), which further generalize results in Drawin $(1968,1969)$, where Thomson's classical theory for electron-atom encounters was applied for collisions between identical particles. The approach is admittedly less than ideal, but unavoidable due to the lack of relevant experimental data and theoretical calculations (see e.g. the

\footnotetext{
${ }^{2}$ http://physics.nist.gov/PhysRefData/ASD/index.html

3 Available at http://ams.astro.univie.ac.at/vald/

${ }^{4}$ http://vizier.u-strasbg.fr/topbase/topbase.html

5 Respectively, a 45- and a 23-level atomic model.

${ }^{6}$ We account for fine splitting also for electron-impact data, by using Barklem's calculations (which originally refer to grouped levels) according to conservation of total rates.
} 

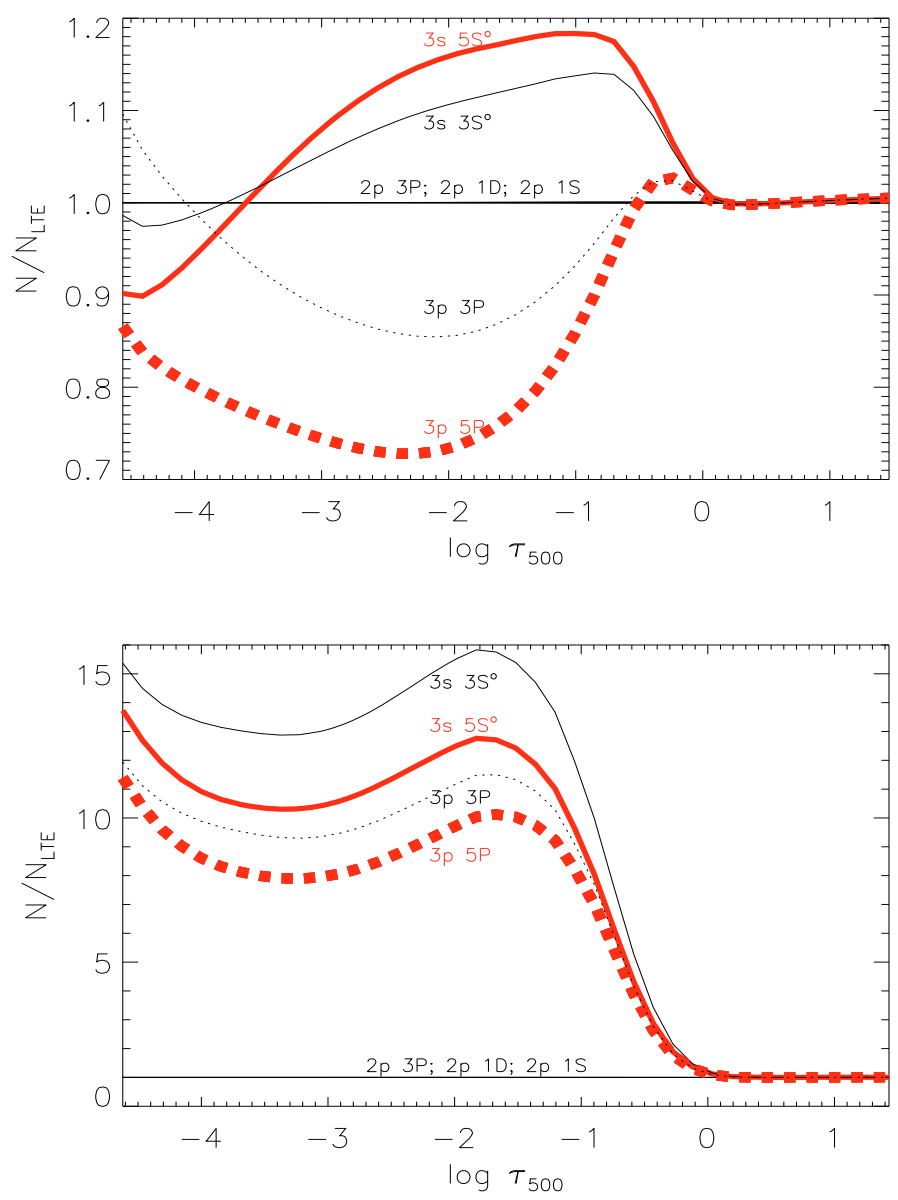

Fig. 2. Departure coefficients in the level population of the lowest energy levels (up to $3 \mathrm{p}^{3} \mathrm{P}$ ) in our oxygen model atom (for the case without $\mathrm{H}$ collisions) are shown respectively for the Sun (upper panel) and a metal-poor turn-off atmospheric model with $[\mathrm{Fe} / \mathrm{H}]=-3$ (lower panel). Note the very different scales for the $y$-axes in the two panels. The thicker lines indicate the departure coefficients for lower and upper levels (solid and dashed curve respectively) in the $777 \mathrm{~nm}$ triplet. Note that only one curve is plotted for the latter levels, because the small differences in the departure coefficients among those fine structure levels does not play an important role here.

discussion in Asplund 2005). In fact, quantum mechanical calculations of rate coefficients exist for only very few electronic transitions of oxygen atoms induced by collisions with $\mathrm{H}$ (Krems et al. 2006; Abrahamsson et al. 2007), and they are unfortunately often of limited applicability for stellar work due to the low range of temperatures explored.

Thus, in order to estimate the size of the main uncertainties in the calculations, we have carried out, similarly to what was done in Fabbian et al. (2006), different non-LTE calculations with a scaling factor $S_{H}$ for transitions between all levels, including for radiatively forbidden transitions (by adopting a minimum $f$-value of $10^{-3}$ in that case).

\section{Results}

\subsection{Non-LTE mechanisms}

The behaviour of the departure coefficients $\beta_{i}$ (defined as the ratio of the populations in non-LTE and LTE, $\beta_{i}=n_{i} / n_{i}^{\mathrm{LTE}}$ ) with atmospheric depth for various energy levels in the atomic model is shown in Fig. 2 for the Sun and a metal-poor turn-off model.
Non-LTE effects for the O I $777 \mathrm{~nm}$ lines at solar metallicity are mainly caused by source function dilution, in the atmospheric layers where the lines form, with respect to the Planck function that represents the LTE expectation. This is due to radiative losses in the triplet itself causing line strengthening compared to LTE. As seen in Fig. 2, the upper level $\left(3 p{ }^{5} \mathrm{P}\right)$ of the transition gets underpopulated, while the lower level $\left(3 \mathrm{~s}{ }^{5} \mathrm{~S}^{\mathrm{o}}\right)$ gets overpopulated. If stimulated emission is neglected, this driving effect can be well described as $S_{1} / B=\beta_{\mathrm{u}} / \beta_{1}$, where $S_{1}$ is the line source function, and $B$ the Planck function. The two-level approximation will give a reasonable description of the non-LTE line formation in the Sun, implying that it is the processes in the transition itself that matter most.

At low metallicity instead, particularly at the highest temperatures explored, that approximation breaks down. The upper level $\left(3 \mathrm{~s}{ }^{3} \mathrm{~S}^{\circ}\right)$ of the $130 \mathrm{~nm}$ O I resonance lines is in effect metastable, since radiative de-excitation will be immediately followed by an excitation (due to large opacity in those lines). Thus, very large overpopulation compared to LTE develops in the level, because of the larger net rate for it than in LTE. This is caused by radiative transitions having this as their lower level (i.e. by photon losses in higher excitation levels), combined with the low rate coefficients out of the level.

The $3 \mathrm{~s}{ }^{3} \mathrm{~S}^{\mathrm{o}}$ overpopulation tends to propagate to the $3 \mathrm{~s}$ ${ }^{5} S^{o}$ level via efficient intersystem collisional coupling, and line strengthening of the $777 \mathrm{~nm}$ lines results, due to much increased line opacity. Given the large energy difference with the ground state, collisions to it from the levels of interest are inefficient in maintaining LTE. The dominant contribution to the non-LTE effect at low metallicity thus comes from line opacity, because the lower level of the IR triplet is strongly overpopulated with respect to LTE (Fig. 2). The non-LTE abundance corrections then become $\sim-0.5$ dex at $[\mathrm{Fe} / \mathrm{H}]=-3$ for a typical metal-poor turnoff halo star, and up to -0.9 dex if neglecting $\mathrm{H}$ collisions.

As seen in Fig. 3, in non-LTE the line formation at very low metallicity is shifted to higher atmospheric layers with respect to LTE. Since the population of the lower level in the oxygen $777 \mathrm{~nm}$ triplet transition rises very steeply with respect to the LTE expectation when moving outward in the atmosphere, the $\beta$ departure coefficient will become large. Figure 3 also shows that the effect (on line-center flux) of photon losses in the line, causing the source function to drop below the Planck function, is small at low $[\mathrm{Fe} / \mathrm{H}]$. The opacity effect dominates, with optical depth unity moving up in the atmosphere because of the overpopulation of the lower level of the transition. The large line opacity at the shallower depths where line formation occurs in non-LTE will thus make the absorption stronger.

Even with fewer free electrons at low metallicity, sufficient intersystem collisional coupling is maintained between levels of similar excitation in the triplet and quintet systems. The $3 \mathrm{~s}^{3} \mathrm{~S}^{\mathrm{o}}$ and the $3 \mathrm{~s}{ }^{5} \mathrm{~S}^{\mathrm{o}}$ levels are in fact very close energetically and, despite the corresponding transition being radiatively forbidden, they are strongly coupled via collisions, which explains why their departure coefficients behave similarly, as seen in Fig. 2. Due to decreased amount of free electrons and to low rates for collisional transitions with larger energy separation, electron collisions are more and more ineffective in driving the line formation closer to LTE via depopulating these two levels via other channels, e.g. to the ground state. The less efficient thermalization via impacts is thus unable to balance the tendency of the triplet levels to overpopulate. In addition, the lower level of the $777 \mathrm{~nm}$ transition is effectively metastable due to negligible net radiative rates to the ground state. 

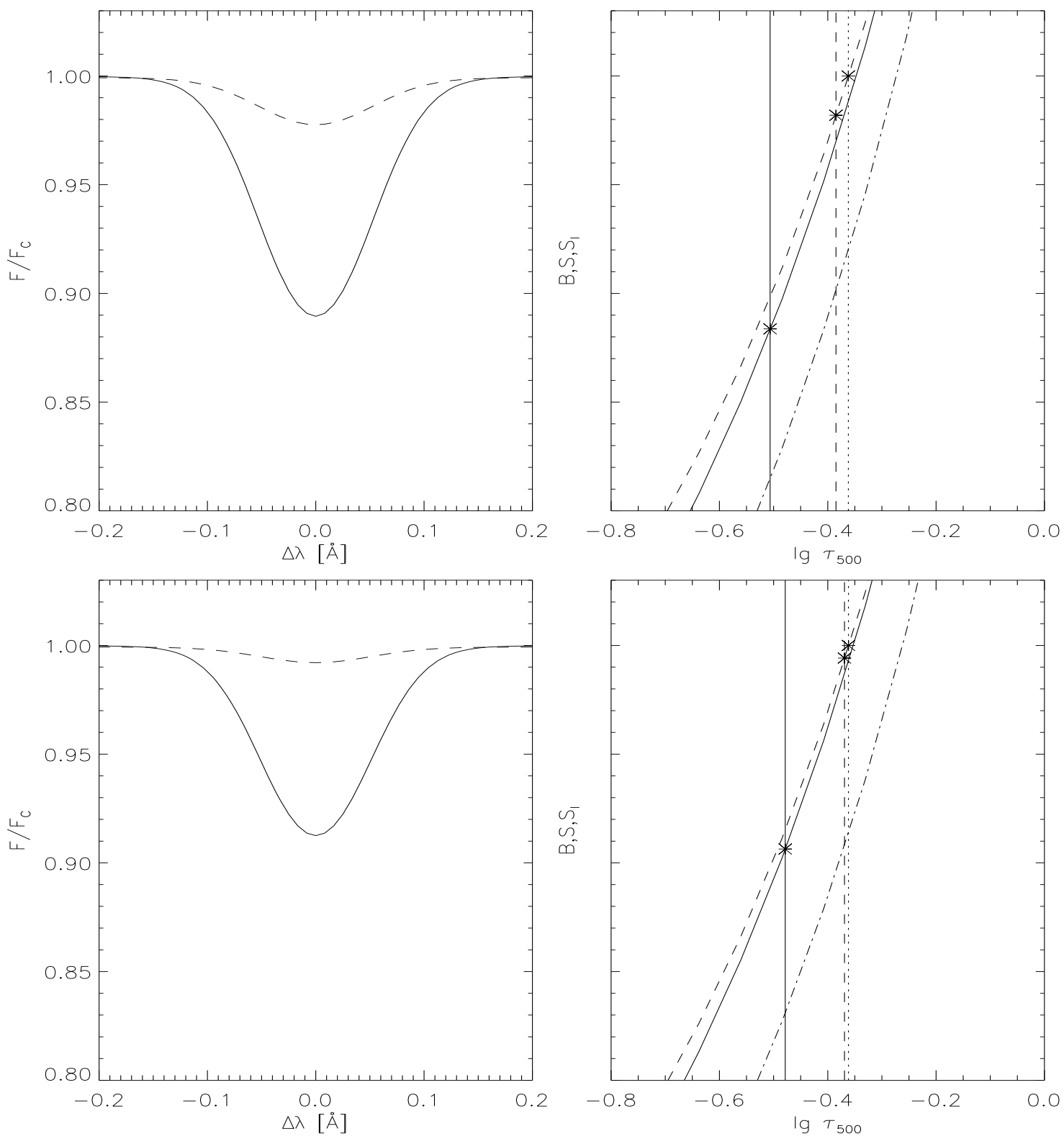

Fig. 3. The oxygen feature at $777.2 \mathrm{~nm}$ (the bluest and strongest of the $\mathrm{O}$ I triplet lines), for $[\mathrm{Fe} / \mathrm{H}]=-3.00$ (top panels) and -3.50 (bottom panels). The atmospheric models have $T_{\text {eff }}=6500 \mathrm{~K}, \log g=4.00[\mathrm{cgs}]$ and solar-scaled, alpha-enhanced $([\alpha / \mathrm{Fe}]=+0.4)$ abundances. The case with no hydrogen collisions is shown here. Top left: the resulting LTE (dashed curve) and non-LTE (solid curve) flux profiles normalized to continuum flux, for the spectral feature considered. Top right: normalized values of $B$ (dashed), $S$ (solid) and $S_{1}$ (dash-dot). The vertical scale in the two cases is therefore the same. The left panel illustrates how at low metallicity the line becomes much stronger in non-LTE compared to LTE. In the right panel, the starred symbols show fluxes corresponding to the Eddington-Barbier formation height, the latter being indicated by vertical dotted, dashed and solid lines (respectively for continuum, LTE line center, and non-LTE line center). Note that the Eddington-Barbier value is $\tau_{v}^{\text {cont }}=2 / 3$ exactly when the source function varies linearly with optical depth, while it is slightly smaller in this case. The right panel shows that at low metallicity the source function effect is small. Bottom: as above, but for $[\mathrm{Fe} / \mathrm{H}]=-3.50$.

Among the IR O I triplet lines, the strongest (bluest) suffers generally more severe non-LTE effects. This is due to it having relatively more important photon losses, and given that it will form further out where departures from LTE are largest. The difference in the estimated abundance correction among the $777 \mathrm{~nm}$ lines usually remains within $0.05 \mathrm{dex}$, but grows towards solar metallicity. The abundance corrections differ by as much as $\sim-0.15$ dex at $T_{\text {eff }}=6500 \mathrm{~K}, \log g=2,[\mathrm{Fe} / \mathrm{H}]=0$, where very large non-LTE effects are found. Thus, adopting the same correction for all three lines is not appropriate, especially in such case, and detailed non-LTE calculations are necessary. This is due to substantially different line formation depths, giving larger non-LTE effects for the line that is formed further out, i.e. the strongest (bluest) one, because of outward increase of the departure coefficients.

Across all of the parameter space explored, the upper level $\left(3 \mathrm{~s}^{3} \mathrm{~S}^{\circ}\right)$ in the OI UV resonance lines tends to become overpopulated. Large radiation excess is maintained in the resonance lines, becoming particularly strong at low metallicity, for high gravity models.

In the $777 \mathrm{~nm}$ lines instead, the ratio of $J$ over $B$ tends to be $<1$ due to photon losses. This is relatively more important 
Table 1. Results (for the strongest triplet line at $777.2 \mathrm{~nm}$ ) obtained using our 54-level atomic model. The non-LTE and LTE equivalent widths are given to show the sensitivity to various mechanisms.

\begin{tabular}{|c|c|c|}
\hline & $\begin{array}{c}W_{\text {non-LTE }} \\
{[\mathrm{m} \AA]}\end{array}$ & $\begin{array}{l}W_{\mathrm{LTE}} \\
{[\mathrm{m} \AA]}\end{array}$ \\
\hline$S_{\mathrm{H}}=\mathbf{0}$ & & \\
\hline standard case & 14.2 & 2.9 \\
\hline$[\mathrm{Fe} / \mathrm{H}]=-3.5$ & 10.9 & 1.0 \\
\hline $\log \epsilon_{\mathrm{O}}=6.0$ & 6.3 & 1.0 \\
\hline$S_{\mathrm{H}}=1$ & & \\
\hline standard case & 6.7 & 2.9 \\
\hline$[\mathrm{Fe} / \mathrm{H}]=-3.5$ & 4.2 & 1.0 \\
\hline $\log \epsilon_{\mathrm{O}}=6.0$ & 2.9 & 1.0 \\
\hline no $130 \mathrm{~nm}$ O I lines & 3.9 & 2.9 \\
\hline no $130 \mathrm{~nm}$, no $136 \mathrm{~nm}$ O I lines & 3.6 & 2.9 \\
\hline $3 \mathrm{~s}-3 \mathrm{~s}$ Barklem $\mathrm{e}^{-}$ & 7.2 & 2.9 \\
\hline all Barklem $\mathrm{e}^{-}$but $3 \mathrm{~s}-3 \mathrm{~s}$ & 6.5 & 2.9 \\
\hline no $3 \mathrm{~s}-3 \mathrm{~s}$ coupling & 5.2 & 2.9 \\
\hline old $\mathrm{e}^{-}$collisions & 6.2 & 2.9 \\
\hline old $\mathrm{e}^{-}$collisions, $3 \mathrm{~s}-3 \mathrm{~s}$ Barklem $\mathrm{e}^{-}$ & 6.4 & 2.9 \\
\hline old $\mathrm{e}^{-}$collisions, no $\mathrm{O}+\mathrm{H} 3 \mathrm{~s}-3 \mathrm{~s}$ & 5.0 & 2.9 \\
\hline no UV background line opacity & 7.8 & 2.9 \\
\hline no H Ly- $\beta$ opacity & 6.8 & 2.9 \\
\hline $\mathrm{Si} * 0.1$ & 9.4 & 2.9 \\
\hline
\end{tabular}

in stars like the Sun, and causes the upper levels in the $777 \mathrm{~nm}$ triplet to depopulate.

Photoionization was found not to be important across the parameter space explored in this study.

\subsubsection{Sensitivity of line formation: main atomic transitions}

In Table 1 we provide the equivalent widths resulting from some of the tests carried out on our 54-level atomic model in order to check the sensitivity of the $777 \mathrm{~nm}$ triplet line formation to various transitions and processes in the atomic model. The standard case shown $\left(T_{\mathrm{eff}}=6500 \mathrm{~K}, \log g=4,[\mathrm{Fe} / \mathrm{H}]=-3, \xi=1\right.$, $\log \epsilon_{\mathrm{O}}=6.5$ ) includes the new electron-impact data by Barklem (2007a). It results in $\Delta \log \epsilon_{\mathrm{O}} \sim-0.9$ and $\sim-0.5$ dex without and with the inclusion of $\mathrm{H}$ collisions respectively. The corresponding non-LTE abundance corrections at $[\mathrm{Fe} / \mathrm{H}]=-3.5$ increase to $\Delta \log \epsilon_{\mathrm{O}} \sim-1.2$ and $\sim-0.9$ dex respectively.

Modifications to the above-mentioned standard case were tested in order to provide a measure of the sensitivity of the nonLTE effects to various mechanisms. The changes for which results are shown in Table 1 are respectively: decrease in metallicity by half dex (to $[\mathrm{Fe} / \mathrm{H}]=-3.5$ ); and decrease in oxygen abundance by half dex (to $\log \epsilon_{\mathrm{O}}=6.00$ ). For the case including $\mathrm{H}$ collisions, additional tests are, following the order in Table 1: removing the $130 \mathrm{~nm}$ O I resonance lines; removing also the intersystem $136 \mathrm{~nm}$ UV O I lines; removing all of the new electron collisions by Barklem except in the radiatively forbidden transition between levels $3 \mathrm{~s}^{3} \mathrm{~S}^{\mathrm{o}}$ and $3 \mathrm{~s}{ }^{5} \mathrm{~S}^{\mathrm{o}}$; including all the new electron collisions by Barklem except the previous one; no $3 \mathrm{~s}{ }^{3} \mathrm{~S}^{\circ}$ and $3 \mathrm{~s}{ }^{5} \mathrm{~S}$ coupling (i.e. removing both electron and $\mathrm{H}$ collisions between those levels); use of old electron collision data from 23level model atom of K93 instead of Barklem's data; same as previous but with new electron collision data between levels $3 \mathrm{~s}^{3} \mathrm{~S}^{\circ}$ and $3 \mathrm{~s}{ }^{5} \mathrm{~S}^{\circ}$; old electron data but no $\mathrm{H}$ collision coupling between the latter levels; neglect of UV background line opacities; exclusion of the opacity contribution by the blending $\mathrm{H}$ Ly- $\beta$ line; and decrease by 1 dex in Si abundance.
From the results shown in Table 1, it is clear that the most important transitions are the O I resonance lines (together with the corresponding UV background opacity), and the intersystem collisional coupling between the $3 \mathrm{~s}^{3} \mathrm{~S}^{\mathrm{o}}$ and $3 \mathrm{~s}{ }^{5} \mathrm{~S}^{\mathrm{o}}$ states. The model atom employed in the standard case is also the one we then used to derive our non-LTE corrections across the whole parameter space (see Sect. 3.2).

We also carried out "multi-MULTI" tests, with results for the case without $\mathrm{H}$ collisions shown in Fig. 4. Through such tests, the relative effect on the O I $777 \mathrm{~nm}$ triplet line strength of multiplying the different rates for radiative and electron collisional transitions individually by a factor of two is revealed. One can thus grasp the impact of different atomic transitions on the formation of the lines of interest. The panels in the left column of Fig. 4 are for radiative rates, those in the right column are for rates due to collisions with electrons. The relative influence of important atomic transitions on the non-LTE line strength of the O I $777 \mathrm{~nm}$ triplet $\left(3 \mathrm{~s}{ }^{5} \mathrm{~S}^{\mathrm{o}}-3 \mathrm{p}{ }^{5} \mathrm{P}\right)$ is shown. The thickness indicates the relative change (as marked in each panel) in nonLTE equivalent width $\left(\mathrm{W}^{\text {pert }}-\mathrm{W}^{\text {ref }}\right) / \mathrm{W}^{\text {ref }}$ between the perturbed and reference case, while the arrows indicate the net flow for each transition (evaluated where $\tau_{\text {line center }}=1.0$ for the $777 \mathrm{~nm}$ triplet). Solid and dashed lines respectively indicate positive (reinforcing) and negative (weakening) contribution from that particular transition to the strength of the line studied.

As seen in Fig. 4, and as found by previous authors (e.g. Kiselman 1991, 1993), the formation of the OI triplet in the Sun is mostly influenced by photon losses in those lines and can be described reasonably well by a two-level approximation. We stress however that our calculations also show an additional contribution from line opacity, due to the lower level of the triplet transition becoming overpopulated. This increases the resulting non-LTE effects for the Sun to a greater amount than usually adopted, up to $\sim-0.3$ dex when neglecting $H$ collisions.

At these solar or moderately low metallicities, the line opacity effect in the OI IR triplet starts to dominate (over that caused by the source function drop) for hot giants. It becomes much more predominant at very low metallicity, where we find an increase in the size of the abundance corrections with increasing $T_{\text {eff }}$ and decreasing $[\mathrm{Fe} / \mathrm{H}]$, related, respectively, to increased level overpopulation via collisions, and to decreasing continuum opacity. An increase in gravity at metallicities below $[\mathrm{Fe} / \mathrm{H}] \sim-2$ will also tend to cause larger non-LTE corrections. This effect is related to the increased sensitivity of the line formation process to intersystem collisional coupling in that regime.

So, while in the Sun both the decrease of the source function below the Planckian and the increase in line opacity are important, with the first being predominant, the metal-poor turnoff model is mostly affected by the latter. The effect of the line source function dilution does not change significantly with $[\mathrm{Fe} / \mathrm{H}]$ at very low metallicity, its contribution to line strengthening remaining $\$ 10 \%$. For parameters typical of metal-poor turn-off stars, we find a trend of increasing non-LTE corrections with decreasing $[\mathrm{Fe} / \mathrm{H}]$. This is caused by the behaviour of continuous opacity at the UV wavelengths of interest (i.e., around the OI $130 \mathrm{~nm}$ features), which decreases substantially (up to $\sim 15 \%$ ) across the line formation layers when the metal content goes from $[\mathrm{Fe} / \mathrm{H}]=-3$ to -3.5 . Even though the contribution of Rayleigh scattering to total continuous opacity is dominant for layers around and above those where the $777 \mathrm{~nm}$ features are formed, it does not vary when metallicity decreases from $[\mathrm{Fe} / \mathrm{H}]=-3$ to -3.5 . Instead, it is the decreased contribution by $\mathrm{Si}$ photoionization to total continuous opacity when $[\mathrm{Fe} / \mathrm{H}]$ 

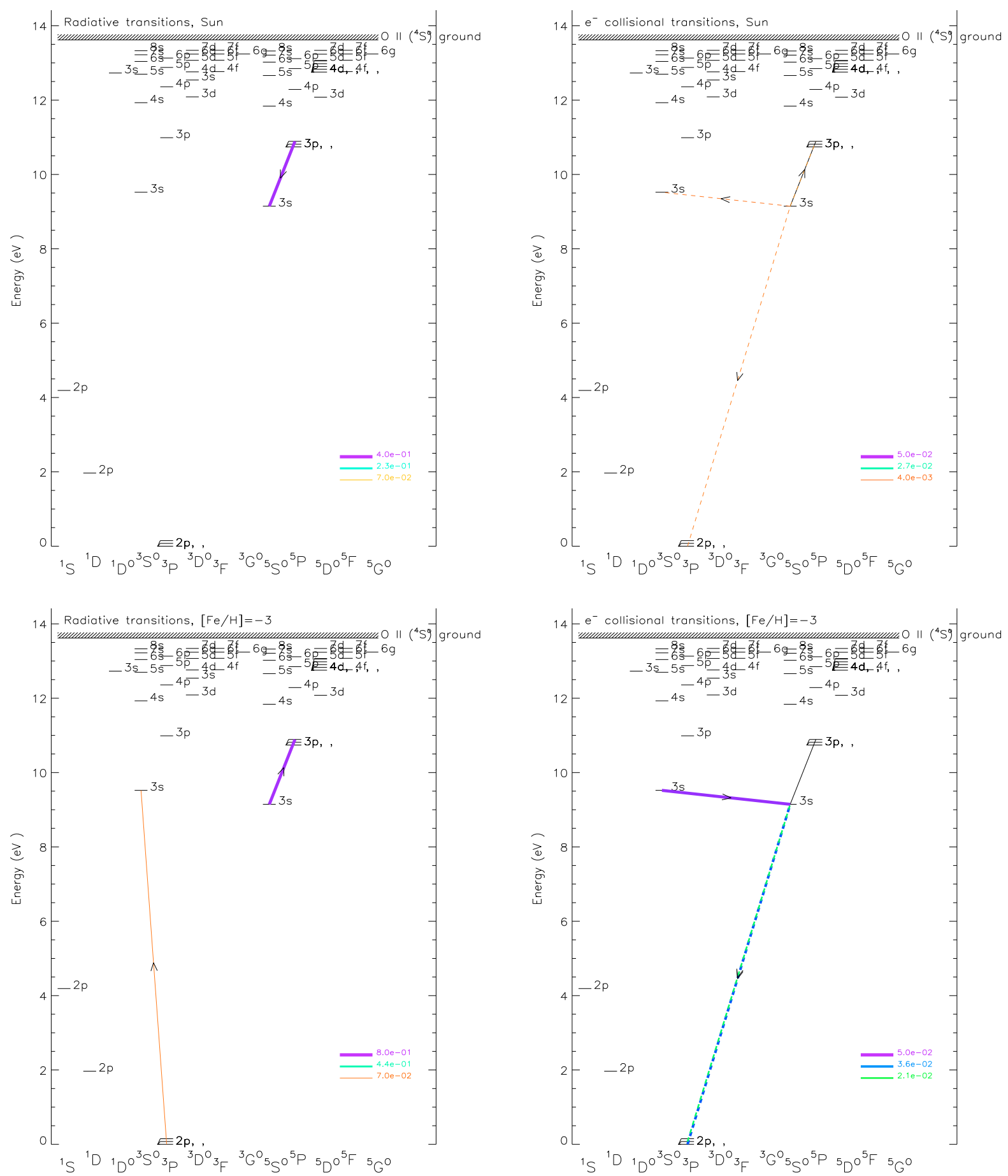

Fig. 4. Results from multi-MULTI runs for a solar model (top panels), and for a star with $T_{\text {eff }}=6500 \mathrm{~K}, \log g=4.00$ and $[\mathrm{Fe} / \mathrm{H}]=-3$ (bottom panels), when neglecting $\mathrm{H}$ collisions.

decreases (see Fig. 5) that deprives the photons in the O I resonance lines of an alternative route. Their destruction probability via the photoionization channel therefore decreases. As a consequence, overpopulation (driven by photon losses in higherexcitation lines) of the upper level in the $\mathrm{O} I$ resonance lines tends to increase. At the same time, the lower level of the $777 \mathrm{~nm}$ transition too will correspondingly become more overpopulated due to efficient intersystem collisional coupling. At low metallicity, the non-LTE effect on the O I $777 \mathrm{~nm}$ triplet feeds on the flow via collisions from the $3 \mathrm{~s}{ }^{3} \mathrm{~S}^{\mathrm{o}}$ state. The level occupation numbers in the lower level of the IR oxygen triplet thus stay closely coupled to the large overpopulation in the upper level of the UV $130 \mathrm{~nm}$ resonance lines, a situation which LTE cannot predict.

At $[\mathrm{Fe} / \mathrm{H}] \sim-3$, the triplet lines become stronger when increasing the radiative rates for the resonance lines (which causes larger overpopulation in level $3 \mathrm{~s}^{3} \mathrm{~S}^{\mathrm{o}}$, and in the lower level of the $777 \mathrm{~nm}$ triplet as a consequence of collisional coupling). Note that in Fig. 4, the net flow (indicated by the direction of the arrows for each transition) is evaluated where $\tau=1$ for the line center of the $777 \mathrm{~nm}$ triplet. This generally gives a good indication of the driving mechanisms. However, at low metallicity, one finds in this way that the radiative rate in the triplet is upward. A check instead reveals that in this case the rates actually change 
sign across the atmosphere. While at $\tau=1.0$ at line center there is a small upward flow, the net rate turns to being downward in deep layers. This last effect dominates, explaining the overall line strengthening marked in the corresponding figure, when increasing the radiative rate in the O I IR triplet. Excluding the transition itself, the most significant impact on the triplet lines at low metallicity is from pumping in the O I $130 \mathrm{~nm}$ resonance lines.

Concerning electron collisions, the main effects at low metallicity are strengthening of the line through relatively efficient triplet-quintet intersystem coupling via electron collisions ${ }^{7}$ between the similarly highly-excited levels $3 \mathrm{~s}{ }^{3} \mathrm{~S}^{\mathrm{o}}$ and $3 \mathrm{~s}{ }^{5} \mathrm{~S}^{\mathrm{o}}-$ which helps to propagate the overpopulation from the former state to the IR oxygen triplet - and line weakening via collisions to the ground state. At low metallicity, both lower $\left(3 \mathrm{~s}{ }^{5} S^{\circ}\right)$ and upper $\left(3 p{ }^{5} \mathrm{P}\right)$ levels of the triplet lines are very overpopulated, but by different amounts.

\subsubsection{Importance of OIUV transitions and background line opacities}

According to our calculations, even in metal-poor stars, at least for frequencies near their quasi-saturated core, the extremely optically thick $130 \mathrm{~nm}$ O I resonance lines $\left(2 \mathrm{p}^{4}{ }^{3} \mathrm{P}_{2,1,0}-3 \mathrm{~s} 3 \mathrm{~S}^{\circ}\right)$ form in the very topmost layers of the photosphere as strong absorption features. Despite this, their thermalization depth lies much deeper in than where monochromatic optical depth in the lines is unity, due to the very large amount of scattering. In particular their $J / B \gg 1$ where the $777 \mathrm{~nm}$ lines form. The properties of scattered photons are decoupled from local conditions, which gives a typical non-LTE situation.

The fact that the O I resonance lines are so optically thick even at low $[\mathrm{Fe} / \mathrm{H}]$ means that at the formation layers of interest, collisional de-excitation from their upper (metastable) level to the lower level of the $777 \mathrm{~nm}$ transition will be favoured with respect to de-excitation to the ground. This situation causes large non-LTE effects at low metallicity. When removing the UV O I resonance lines, the non-LTE effects remain important (see Table 1) due to photon losses from higher levels.

Previous studies (e.g., K93; Gratton et al. 1999) found that UV radiation should not play a major role in the formation of the IR oxygen triplet in very-metal poor stars. They were, however, lacking the detailed quantum mechanical calculations for intersystem electron collisions which we employ here and which cause increased non-LTE effects.

Background absorption from UV lines of other elements mitigates (by providing an escape route other than collisional de-excitation and collisional transfer to the quintet system) but does not extinguish this effect. The exact amount of boundfree absorption from different metals is however still debated (e.g. Balachandran \& Bell 1998; Asplund 2004), due to the possibility that continuum rates, e.g. for Fe I, may be higher than what is usually adopted. As noted by Allende Prieto et al. (2003a,b), predicting a realistic flux in the UV is more complicated than for visible or IR wavelengths. The knowledge of

\footnotetext{
7 As seen in Fig. 4, this channel gives instead a weakening effect in the Sun. The reason is clear from the previously discussed Fig. 2 which shows that in the Sun, the departure coefficient is larger for $3 \mathrm{~s}{ }^{5} \mathrm{~S}^{\circ}$ (lower level of $777 \mathrm{~nm}$ transition, in the quintet system) than for $3 \mathrm{~s}{ }^{3} \mathrm{~S}^{\circ}$ (upper level of O I $130 \mathrm{~nm}$ UV resonance lines, in the triplet system). An increase of this collisional coupling channel in the Sun will therefore decrease the overpopulation and thus the opacity effect for the $777 \mathrm{~nm}$ transitions, making those lines form deeper in, where smaller non-LTE effects are felt.
}

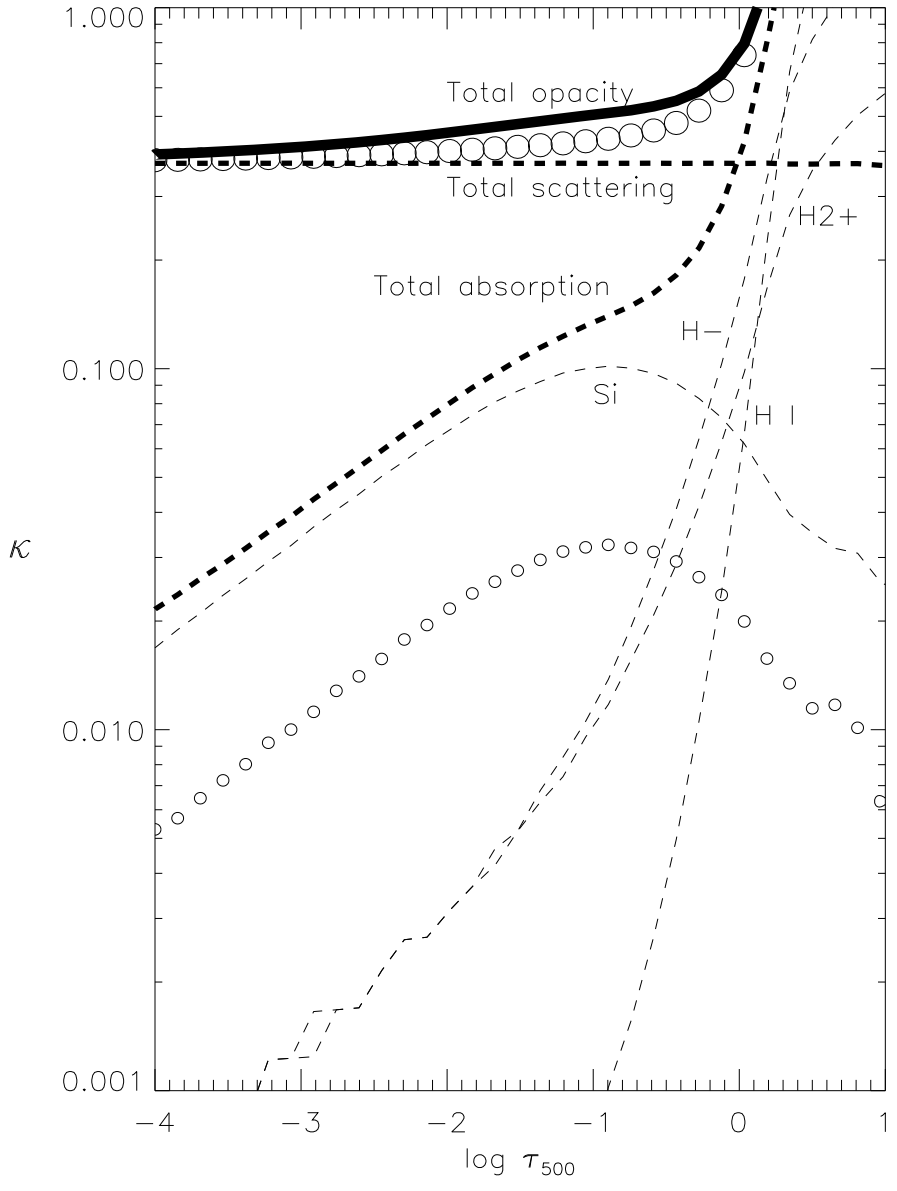

Fig. 5. Contributions to total continuous opacity around the $130 \mathrm{~nm} \mathrm{O} \mathrm{I}$ lines for a $T_{\text {eff }}=6500, \log g=4,[\mathrm{Fe} / \mathrm{H}]=-3$ model atmosphere. The absorption and the scattering part of total opacity are shown by the two thick dashed curves, as marked. For absorption, the importance of its main components $\left(\mathrm{Si}, \mathrm{H}^{-}, \mathrm{H}_{2}^{+}\right.$and $\left.\mathrm{H} \mathrm{I}\right)$ is depicted. The total amount of continuous opacity is shown by the thick solid curve. For comparison, we also show (small circles) the reduced contribution of $\mathrm{Si}$ at $[\mathrm{Fe} / \mathrm{H}]=-3.5$ and consequent reduced total continuous opacity (large circles). The rest of the sources of opacity in the figure do not change significantly with this decrease in metallicity.

accurate bound-bound and photoionization cross-sections and thresholds for metals becomes important, with a large quantity of accurate atomic data required for modeling. Below $\sim 1700 \AA$, Si I bound-free absorption plays an important role. Rayleigh scattering by hydrogen atoms is also important in the ultraviolet for cool stars, in particular at low metallicity. In addition to boundfree metal edges, a sharp increase in opacity is expected at short UV wavelengths, also due to the haze caused by background line absorption. Neglecting these effects will seriously overestimate the UV radiation field within the upper atmospheric layers of late-type stars.

Figure 5 shows the main contributions to total continuous opacity for UV wavelengths around the $\mathrm{O}$ I resonance lines and a MARCS model having $T_{\text {eff }}=6500 \mathrm{~K}, \log g=4,[\mathrm{Fe} / \mathrm{H}]=-3$ and $\log \mathrm{O}=6.5$. As seen, scattering is roughly constant across the atmospheric model. Its contribution to total opacity dominates down to the atmospheric layers $\left(\log \tau_{500} \sim-0.5\right)$ where the IR oxygen triplet lines form. The contribution of absorption, even though smaller, is still significant at such layers predominantly thanks to $\mathrm{Si}$ I bound-free edges. It is this component which proves important to understand the trends of the nonLTE corrections at low metallicity, since it is the only one that 
varies significantly with $[\mathrm{Fe} / \mathrm{H}]$. At $[\mathrm{Fe} / \mathrm{H}]=-3.5$, its contribution to absorption for the atmospheric layers of interest reduces to $\sim 35 \%$ of the value at $[\mathrm{Fe} / \mathrm{H}]=-3$. Thus, total continuous opacity becomes significantly smaller. No other metal component plays a significant role. Deeper in, absorption takes over, due to $\mathrm{H}^{-}$and $\mathrm{H}_{2}^{+}$at first, and then to the rapid increase of neutral hydrogen photoionization.

The greater continuum absorption at $[\mathrm{Fe} / \mathrm{H}]=-3$ compared to $[\mathrm{Fe} / \mathrm{H}]=-3.5$ reduces the overpopulation in the upper state of the UV O I resonance transitions because it provides an alternative route to photons in those lines (absorption by the continuum instead of by the line itself).

The non-LTE corrections are found to be very sensitive to the UV radiation field. Thus, background opacity caused by lines of other elements has been accounted for in the case of important transitions, in particular the contribution of $\mathrm{H} \mathrm{Ly}-\beta$ and of features around the O I $130 \mathrm{~nm}$ resonance lines. The latter inclusion has proven particularly important because it significantly reduces the otherwise even more extreme non-LTE effects (see Table 1). In total, 151 lines from VALD were used, around the 130,103 and $136 \mathrm{~nm}_{\text {transitions }}^{8}$. For these background features, the line source function was assumed to be equal to the Planck function. This approximation is sufficient also to describe the case of pumping by hydrogen $\mathrm{Ly}-\beta$ radiation. This was tested by solving the radiation transfer problem for the hydrogen atom and taking the $\mathrm{J}$ field as input for the non-LTE calculations on oxygen. The results showed only very small differences with respect to our approximation, for the formation of the oxygen triplet lines of interest here. Note that $\mathrm{H} \mathrm{Ly}-\beta$ gives the single greatest contribution to background line opacity, however it is the total contribution of the several blending metal lines falling around $130 \mathrm{~nm}$ which is most important, in particular due to S I $130.23 \mathrm{~nm}$, which falls close to line center of the bluest O I resonance line, Si II $130.44 \mathrm{~nm}$, which is located in the blue wing of the central resonance line but also affects the $130.22 \mathrm{~nm} \mathrm{O} \mathrm{I}$ line by depressing the continuum, and S I $130.59 \mathrm{~nm}$, which falls close to the center of the reddest resonance line. In addition, a number of lines of $\mathrm{Fe}, \mathrm{Ca}, \mathrm{P}$ and other elements give a smaller contribution to line haze. The lines around the O I UV intersystem lines are instead not important.

Our study reveals that the inclusion of the UV opacity contribution does not have a significant impact on the $777 \mathrm{~nm}$ triplet lines in the Sun, because the non-LTE effect is in that case at least in good part controlled by processes in those same IR lines.

\subsubsection{Effects of collisions with electrons and hydrogen}

Figure 6 shows the net rates at low metallicity for the highexcitation levels $3 \mathrm{~s}{ }^{5} \mathrm{~S}^{\mathrm{o}}$ and $3 \mathrm{~s}{ }^{3} \mathrm{~S}^{\mathrm{o}}$. The figure clearly illustrates how the balance in the population of the lower level of the $777 \mathrm{~nm}$ oxygen triplet is mainly determined by incoming flow due to collisional coupling - which transmits the overpopulation present in the $3 \mathrm{~s}^{3} \mathrm{~S}^{\mathrm{o}}$ state - moderated by radiative excitation in the IR lines.

We have included in our model atom a homogeneous set of electron-impact excitation data, thanks to the availability of recent theoretical quantum mechanical calculations (Barklem 2007a). Table 1 shows the results of testing various modifications (including for electron collisions) to the atomic model. The effect of the new electron-impact data is to give larger non-LTE

\footnotetext{
${ }^{8}$ Respectively accounting for, in order of decreasing importance, the effect of features around the resonance lines, of absorption due to $\mathrm{H}$ Ly- $\beta$, and of lines falling close to the intersystem oxygen lines.
}

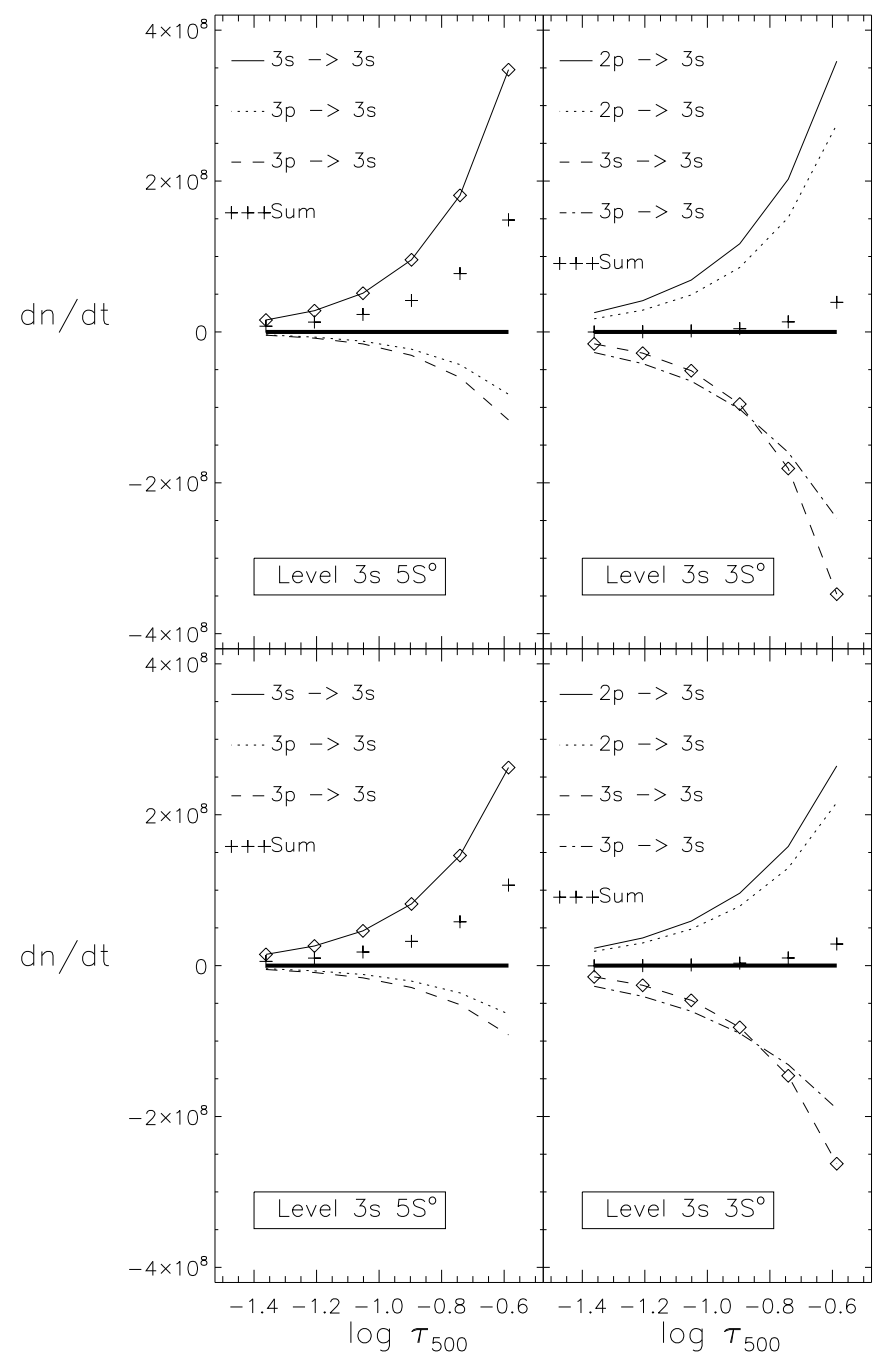

Fig. 6. Net rates contributing to the balance of levels $3 \mathrm{~s}{ }^{5} \mathrm{~S}^{\mathrm{o}}$ (left) and $3 \mathrm{~s}{ }^{3} \mathrm{~S}^{\mathrm{o}}(r i g h t)$. Lines with and without diamond symbols indicate the contribution to a given rate from collisions, and that from radiation, respectively. Only the rates with large influence on the level population are shown (their summed contribution is represented by crosses). The thick horizontal line at $\mathrm{d} n / \mathrm{d} t=0$ marks the statistical equilibrium expectation that the total sum of all rates to and from a given level is equal to zero. The atmospheric model has $T_{\text {eff }}=6500, \log g=4$ and $\log \epsilon_{\mathrm{O}}=6.5$. Plots are for $[\mathrm{Fe} / \mathrm{H}]=-3$ (upper panels) and -3.5 (lower panels) respectively.

corrections due to increased intersystem coupling (in particular in the $3 \mathrm{~s}^{3} \mathrm{~S}^{\mathrm{o}}-3 \mathrm{~s}{ }^{5} \mathrm{~S}^{\mathrm{o}}$ transition). This is an efficient channel due to its large cross-section (it is a spin-flip transition induced by an exchange interaction of the electrons) and its small $(\sim 0.38 \mathrm{eV})$ energy separation (thus low threshold).

Inelastic collisions with neutral $\mathrm{H}$ atoms as modelled here can play a crucial role at low metallicity, where the scarcity of free electrons means that rates due to $\mathrm{H}$ collisions can become important in late-type stars due to the large $\mathrm{H}$ population density. Under the assumption that van Regemorter's and Drawin's formulae well reproduce the thermalization due to electron and $\mathrm{H}$ collisions respectively, one derives that the ratio of the rates due to the two processes is a function of the energy separation between the levels involved and of temperature (Asplund 2005). These collisions can thus become particularly efficient for levels that are very close in energy. Unfortunately, as already observed by Steenbock \& Holweger (1984), Drawin's formula 
allows at best an order-of-magnitude estimate of the importance of $\mathrm{H}$ collisions. Significant differences have been found compared to more reliable estimates (e.g. experimental data by Fleck et al. 1991; calculations for $\mathrm{Na}$ by Belyaev et al. 1999; for Li by Belyaev \& Barklem 2003; and for $\mathrm{H}+\mathrm{H}$ by Barklem 2007b), differing by up to six orders of magnitude for some transitions. The uncertainty on the efficiency of $\mathrm{H}$ collisions has a significant impact on non-LTE studies. There is some evidence (Allende Prieto et al. 2004) that for oxygen, relatively efficient collisions with $\mathrm{H}$ need to be included in statistical equilibrium calculations. We confirm the impact of their thermalizing effect, especially at low $[\mathrm{Fe} / \mathrm{H}]$ (see Table 1). They tend to mitigate the large overpopulation in the lower level of the $777 \mathrm{~nm}$ triplet and thus reduce the line opacity. The non-LTE abundance corrections at low metallicity become considerably smaller (by $\gtrsim 50 \%$ ).

In the absence of more reliable calculations, the only possibility to obtain an indication of the influence of $\mathrm{H}$ collisions has been to adopt the Drawin recipe. Even though likely not realistic, this may be useful in the case of oxygen, at least (inevitably) as a first approach, to investigate the impact of $\mathrm{H}$ collision efficiency. Given available evidence pointing towards the Drawin formula generally overestimating rates for individual transitions, the range of $S_{\mathrm{H}}=0-1$ adopted here would seem reasonable for our tests, and may be expected to give an idea of the uncertainties involved with respect to stellar abundance estimates from the $777 \mathrm{~nm}$ O I triplet.

We also tested the effect of including the recent data by Krems et al. (2006). Even though their estimated rates (using formula 10 in their paper) are higher than our adopted values, they did not have a significant impact on our results since the transition they consider in the singlet and triplet systems $\left({ }^{1} \mathrm{D}-\right.$ ${ }^{3} \mathrm{P}$ ) involves levels that follow LTE anyway.

\subsection{Overall non-LTE effects across the parameter space}

The high-excitation triplet lines are particularly sensitive to temperature and become much stronger, both in LTE and non-LTE, for larger $T_{\text {eff }}$ values, because the corresponding level population increases, i.e. there are more atoms with electrons in the proper energy level to produce the spectral lines. Surface gravity (pressure) controls the amount of recombination via the Saha equation, but also the relative importance of collisional processes with respect to radiative ones. The lines get stronger in lower gravity models. Metal content - i.e. smaller or larger number of absorbers - also controls the strength of the lines.

An IDL routine ${ }^{9}$ has been prepared to interpolate among the grid, therefore making it possible to calculate detailed abundance corrections for any given star with parameters in the range covered.

The non-LTE line strength is larger than in LTE across the parameter space explored, i.e., negative abundance corrections are found. Figure 7 shows to what extent the strongest (bluest) O I triplet line at $777.19 \mathrm{~nm}$ is affected by the resulting nonLTE corrections for some of the models (typical dwarf, typical turn-off star, and RR Lyrae star). A qualitatively similar trend is seen, namely of decreasing abundance corrections for all models from solar metallicity to $[\mathrm{Fe} / \mathrm{H}]=-2$ and then (except

\footnotetext{
9 The routine and associated data are made available for general use. They are downloadable either as individual files, or as a single compressed file, from ftp://nlte:c1o1nlte@ftp.iac.es (at this same address the routine for carbon non-LTE corrections from Fabbian et al. 2006, is also available).
}

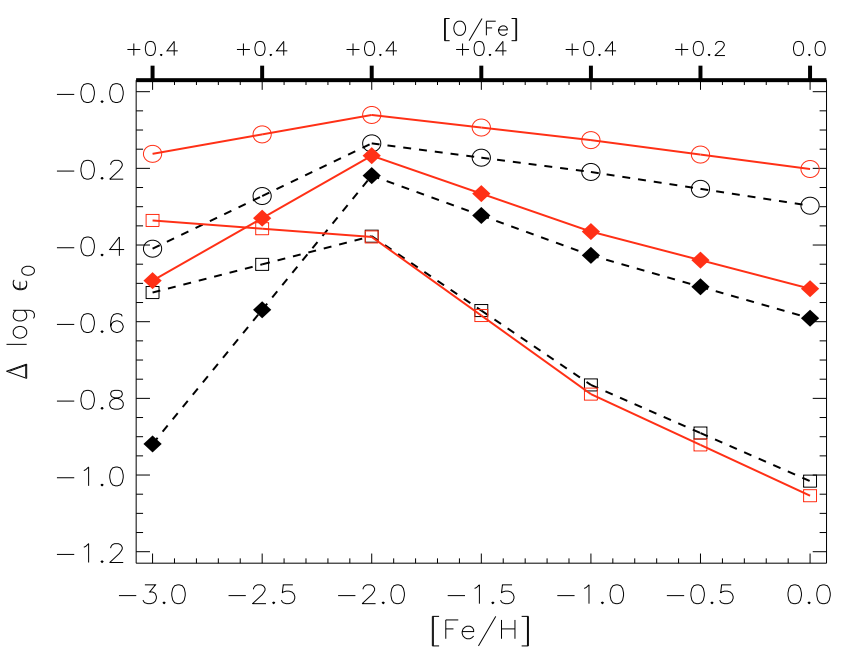

Fig. 7. Non-LTE abundance corrections versus metallicity, for the strongest OI IR $777 \mathrm{~nm}$ triplet line. The lines connect models with given $T_{\text {eff }}$ and $\log g$ but varying metallicity. Different symbols denote, respectively, results for models representative of a normal dwarf ( $T_{\text {eff }}=5780 \mathrm{~K}, \log g=4.44$, indicated by open circles), a normal turnoff star ( $T_{\text {eff }}=6500 \mathrm{~K}, \log g=4$, marked with filled diamonds) and a RR-Lyrae $\operatorname{star}\left(T_{\text {eff }}=6500 \mathrm{~K}, \log g=2\right.$, indicated by open squares). Dashed lines are for no $\mathrm{H}$ collisions, solid lines for a choice of $S_{\mathrm{H}}=1$. Solar-scaled oxygen abundance with $\alpha$-enhancement below solar metallicity was adopted in the calculations, as indicated by the horizontal axis at the top of the figure.

when including $\mathrm{H}$ collisions in the RR Lyrae case) of increasingly severe non-LTE corrections towards very low metallicity, in particular for turn-off stars, where the resulting $\left|\Delta \log \epsilon_{\mathrm{O}}\right|$ reaches $\sim-0.9$ dex at $[\mathrm{Fe} / \mathrm{H}]=-3$, when neglecting $\mathrm{H}$ collisions. This reduces to $\sim-0.5$ dex if $\mathrm{H}$ collisions are included. For the atmospheric models considered, non-LTE corrections are less severe around $[\mathrm{Fe} / \mathrm{H}]=-2$, but still remain significant, reaching $\sim-0.4$ dex for the RR Lyrae star. Interestingly, especially for the latter, $\mathrm{H}$ collisions do not have much impact (the difference staying within 0.1 dex for all models) unless $[\mathrm{Fe} / \mathrm{H}]<-2$. At higher metallicity, including $\mathrm{H}$ collisions in the RR Lyrae case actually makes the non-LTE abundance corrections ever so slightly more severe $^{10}$. For the Sun, non-LTE corrections are -0.3 dex or less severe. In the RR Lyrae case, the abundance corrections at solar metallicity are very large and $\sim-1$ dex.

At metallicities $-2<[\mathrm{Fe} / \mathrm{H}]<0$, the line source function roughly follows a two-level approximation and thus the non-LTE corrections will depend on line strength. This explains their increase in atmospheric models having higher $T_{\text {eff }}$ and $[\mathrm{Fe} / \mathrm{H}]$, and lower $\log g$ (see Fig. 8).

In a more general sense (see Fig. 8, where we show our resulting non-LTE corrections for a range of atmospheric parameters across the grid explored), the abundance corrections increase in size for higher effective temperature atmospheric models due to increased photon losses and/or level overpopulation via collisions. Corrections are small for $T_{\text {eff }} \leq 5500 \mathrm{~K}$, except for solarmetallicity giants, where they can reach $\sim-0.6$ dex. The trend

\footnotetext{
10 Some authors have suggested to calibrate $\mathrm{H}$ collisions on such stars (Clementini et al. 1995; Gratton et al. 1999). Even though RR Lyrae may be prone to very large non-LTE effects, if adopting this strategy, it appears from our results that it would be best to calibrate on metalpoor turn-off stars, where the sensitivity to the choice of $\mathrm{H}$ collisions is higher.
} 

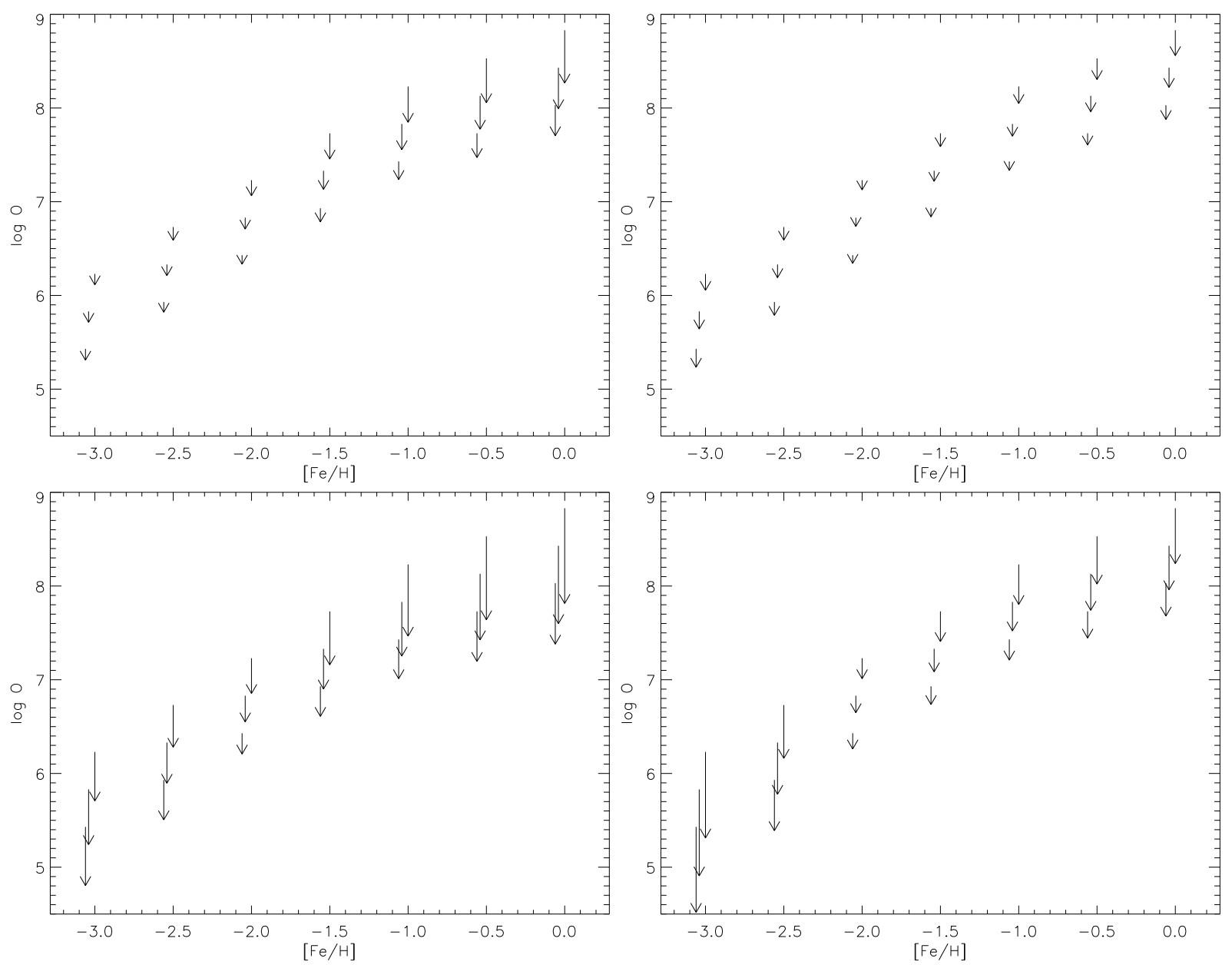

Fig. 8. Non-LTE abundance corrections versus metallicity, for the strongest O I IR $777 \mathrm{~nm}$ triplet line. Top: $T_{\text {eff }}=5500 \mathrm{~K}$; $\log g=2$ (left panel) and $\log g=4$ (right panel) respectively. Bottom: same, but for $T_{\text {eff }}=6500 \mathrm{~K}$. Results shown in all four panels are computed neglecting collisions with $\mathrm{H}$ atoms. The non-LTE corrections tend to become large at high temperature, both in dwarfs and giants. They are significant also in cooler solar-metallicity giants.

with $\log g$ and $[\mathrm{Fe} / \mathrm{H}]$ is more complex. For higher metallicity models, the non-LTE line source function drop due to photon losses has a two-level nature, therefore naturally increasing for stronger lines. Thus, corrections tend to become larger with increasing temperature and metallicity/oxygen abundance and with decreasing $\log g$ in this higher metallicity range. Below $[\mathrm{Fe} / \mathrm{H}] \sim-2$ however, the influence of $\log g$ and $[\mathrm{Fe} / \mathrm{H}]$ is the opposite. The non-LTE corrections tend to increase strongly towards very low metallicity and increasing gravity, suggesting that line formation is controlled by a distinct non-LTE mechanism compared to the higher metallicity regime.

Even though the $777 \mathrm{~nm}$ triplet lines get much weaker towards low metallicity, departures from LTE tend to increase, becoming very large for typical metal-poor turn-off stars. An increase in gravity at low metallicity tends to make the abundance corrections larger, by increasing the collisional rate in the important intersystem coupling channel. As mentioned in Sect. 3.1.2, the non-LTE effect is controlled by Si I photoionization in the continuum. Thus, when the other parameters $\left(T_{\mathrm{eff}}, \log g, \xi\right.$ and $\log \epsilon_{\mathrm{O}}$ ) in the atmospheric model are kept constant, decreasing metallicity alone results in increasing non-LTE effects because of smaller continuum opacity and generally less efficient collisions (smaller contribution to free electron pool). For example, even when adopting efficient $\mathrm{H}$ collisions $\left(S_{\mathrm{H}}=1\right)$, the abundance corrections jump from $\sim-0.5$ to $\sim-0.85$ dex in a model with $T_{\text {eff }}=6500 \mathrm{~K}, \log g=4$ and $\log \epsilon_{\mathrm{O}}=6.5$, when moving from $[\mathrm{Fe} / \mathrm{H}]=-3$ to -3.5 . Finally, oxygen abundance, while controlling the strength of the lines, does not have a crucial influence on the abundance corrections, with differences in $\Delta \log \epsilon_{\mathrm{O}}$ remaining within $\sim 0.1$ dex for a change of $0.5 \mathrm{dex}$ in $\log \epsilon_{\mathrm{O}}$.

In summary, the non-LTE effects are particularly significant at the highest temperatures, in particular for low-gravity, solarmetallicity, and for high-gravity, very low metallicity models. In both cases, when $\mathrm{H}$ collisions are neglected, the non-LTE abundance corrections reach up to $\left|\Delta \log \epsilon_{\mathrm{O}}\right| \sim 1$ dex. The inclusion of collisions with hydrogen atoms between all energy levels generally reduces the effects substantially (several tenths of a dex) at low metallicity, while at higher metallicity it has a smaller impact, due to radiative losses in the line itself being mostly important. The effects amount to -0.3 dex or less in the Sun, but are -0.4 dex or larger for hotter, solar-metallicity stars like Procyon.

In addition, we have tested the influence of using ATLAS9 model atmospheres from Castelli \& Kurucz (2004) without "convective overshooting", instead of MARCS models. The temperature stratification in the MARCS and ATLAS models is generally thought to be quite similar in this parameter space (Gustafsson et al. 2008), so that one would expect the resulting non-LTE corrections to be not too different in magnitude. Any uncertainty from such supposedly small differences should be much less than that due to, say, choice of $\mathrm{H}$ collision efficiency or use of the 1D approximation. We carried out a 
Table 2. Comparison of results obtained with representative cases from two different model atmosphere sets (ATLAS, and MARCS), assuming a solar oxygen abundance of $\log \epsilon_{\mathrm{O}}=8.66$ (Asplund et al. 2004).

\begin{tabular}{lccc}
\hline \hline$T_{\text {eff }}(\mathrm{K}) \backslash \log g$ & $5500 \backslash 4.5$ & $6500 \backslash 2.0$ & $6500 \backslash 4.0$ \\
\hline & $\begin{array}{c}\Delta \log \epsilon_{\mathrm{O}} \\
\text { (ATLAS) }\end{array}$ & $\begin{array}{c}\Delta \log \epsilon_{\mathrm{O}} \\
\text { (ATLAS) }\end{array}$ & $\begin{array}{c}\Delta \log \epsilon_{\mathrm{O}} \\
\text { (ATLAS) }\end{array}$ \\
\hline$[\mathrm{Fe} / \mathrm{H}]=0.0$ & $-0.20 /-0.11$ & $-0.83 /-0.86$ & $-0.53 /-0.45$ \\
{$[\mathrm{Fe} / \mathrm{H}]=-1.0$} & $-0.14 /-0.05$ & $-0.64 /-0.67$ & $-0.36 /-0.31$ \\
{$[\mathrm{Fe} / \mathrm{H}]=-2.0$} & $-0.11 /-0.03$ & $-0.32 /-0.35$ & $-0.20 /-0.15$ \\
{$[\mathrm{Fe} / \mathrm{H}]=-2.5$} & $-0.12 /-0.03$ & $-0.28 /-0.26$ & $-0.27 /-0.16$ \\
{$[\mathrm{Fe} / \mathrm{H}]=-3.5$} & $-0.43 /-0.10$ & $-0.26 /-0.18$ & $-0.87 /-0.54$ \\
\hline & $\Delta \log \epsilon_{\mathrm{O}}$ & $\Delta \log \epsilon_{\mathrm{O}}$ & $\Delta \log \epsilon_{\mathrm{O}}$ \\
& (MARCS) & (MARCS) & (MARCS) \\
\hline$[\mathrm{Fe} / \mathrm{H}]=0.0$ & $-0.18 /-0.10$ & $-0.88 /-0.92$ & $-0.50 /-0.44$ \\
{$[\mathrm{Fe} / \mathrm{H}]=-1.0$} & $-0.12 /-0.05$ & $-0.63 /-0.66$ & $-0.34 /-0.29$ \\
{$[\mathrm{Fe} / \mathrm{H}]=-2.0$} & $-0.11 /-0.03$ & $-0.30 /-0.31$ & $-0.19 /-0.14$ \\
{$[\mathrm{Fe} / \mathrm{H}]=-2.5$} & $-0.16 /-0.04$ & $-0.42 /-0.33$ & $-0.56 /-0.34$ \\
{$[\mathrm{Fe} / \mathrm{H}]=-3.5$} & $-0.52 /-0.14$ & $-0.88 /-0.53$ & $-1.21 /-0.85$ \\
\hline
\end{tabular}

number of test calculations which show that, when using Castelli \& Kurucz atmospheric models describing the Sun and stars of intermediately low metallicity, the resulting non-LTE abundance corrections remain in fact very similar (within a few hundredths of a dex) to those obtained using corresponding MARCS models. The results for the most metal-poor cases reveal that for $[\mathrm{Fe} / \mathrm{H}] \leq-2.5$, the non-LTE abundance corrections obtained at higher gravity become more significant with decreasing metallicity also for ATLAS model atmospheres. Table 2 lists the nonLTE corrections for the central line in the O I IR triplet, obtained with the two sets of models (using our 54-level atomic model, respectively excluding and including the contribution of $\mathrm{H}$ collisions, i.e. $\left.S_{\mathrm{H}}=0 / 1\right)$. The adopted $\alpha$-element abundance for all atmospheric models below solar metallicity in Table 2 is enhanced by +0.4 dex with respect to solar.

Compared to the MARCS case, the ATLAS non-LTE corrections, although still very significant, tend to be less dramatic. The largest discrepancy between results using metal-poor MARCS and ATLAS models is found at low gravity. For both sets of atmospheric models, an LTE description of the O I IR triplet is particularly unrealistic for the lowest-metallicity $([\mathrm{Fe} / \mathrm{H}]=-3.5)$ dwarf case at $T_{\text {eff }}=6500 \mathrm{~K}$.

Knowledge of how the two sets of models compare allows us to include in the next section a discussion of our results in relation to other studies which have employed ATLAS model atmospheres. Since it is almost impossible to disentagle and remove all the different systematics, often due to lack of sufficient information in previous works, we have chosen to compare our results taken at face value with other non-LTE studies in the literature, and then to apply our results directly to relevant existing observations.

A more detailed study comparing oxygen abundance corrections obtained with different sets of model atmospheres, should however be carried out, in particular at very low metallicity, where our tests reveal that when using Castelli \& Kurucz models, the non-LTE effects are less severe compared to results obtained using MARCS models. Within the parameter space explored for the two sets of models, the largest differences (several tenths of a dex when neglecting $\mathrm{H}$ collisions) between the $\mathrm{O}$ I $777 \mathrm{~nm}$ triplet non-LTE corrections appear at the lowest metallicities, both for giant and dwarf models.

The smaller departure coefficients in the very low-metallicity Castelli \& Kurucz models are caused by reduced intersystem coupling (lower rates) via electron collisions, and thus less severe atomic level overpopulation. This is likely due to the effect of existing differences in electron density/temperature stratification in deep atmospheric layers (for example, up to around $400 \mathrm{~K}$ at $[\mathrm{Fe} / \mathrm{H}]=-2.5)$, between the MARCS and ATLAS sets of very metal-poor models. In this context, the failure of the Kurucz models to provide matching oxygen abundances from the near-IR triplet and the forbidden line has previously been attributed to inconsistencies in electron density and temperature gradients of those models near the continuum-forming layers (Israelian et al. 2004). Compared to the MARCS models, we find that the effect of increasingly severe non-LTE corrections with decreasing metallicity starts to appear at lower metallicity in the Castelli \& Kurucz models (for example, reaching $\Delta \log \epsilon_{\mathrm{O}} \sim-0.7 /-0.9 \mathrm{dex}$ with/without $\mathrm{H}$ collisions, for a $T_{\text {eff }}=6500 \mathrm{~K}, \log g=4$ and $[\mathrm{Fe} / \mathrm{H}]=-4$ model $)$. We can only speculate that the two sets of models behave differently due to differences in opacities, and/or equation of state, and/or convection treatment. The issue of which set of 1D models is more suitable or realistic is still open (see discussion in Gustafsson et al. 2008) and of course, further improvements in the modeling (e.g. use of three-dimensional model atmospheres) may prove crucial.

Note that there will be systematic errors when applying, as is often done, corrections computed for one set of model atmospheres, to LTE results obtained with a different set of models, for example due to differences between continuous opacities and ionization balances used in the construction of the model atmospheres and those employed for the non-LTE calculations. Therefore, the abundance corrections we derived in this paper should, strictly speaking, be applied only to results obtained with MARCS model atmospheres. However, given the above discussion, we suggest that they can also be safely applied to LTE abundances from Kurucz models down to intermediately low metallicity.

\section{Comparison with other theoretical non-LTE studies for oxygen}

We now discuss our results in relation to those of previous work present in the literature.

\subsection{Kiselman 1991, 1993, 2001; and related works}

Kiselman (1991, 1993, 2001) has studied and reviewed the nonLTE effects on oxygen lines. A comparison with those works, and with CJ93, Nissen et al. (2002), Akerman et al. (2004), and García Pérez et al. (2006) is of significance. Our results are also relevant to the publications by Israelian et al. (2004), Shchukina et al. (2005) and Meléndez et al. (2006), where the various authors used atomic data based on CJ93. In fact, all the works mentioned in this subsection are at least in part based on the (14- and 45-level) atomic models in that study.

The CJ93 atomic models include estimates by van Regemorter (1962) and the impact approximation of Seaton (1962) for collisions involving radiatively allowed bound-bound transitions, and data available from various authors for radiatively forbidden transitions connecting to the ground state and to the continuum. Collisional coupling between the singlet, triplet and quintet systems is largely absent in those models due to lack of data for several transitions, in particular, for intersystem coupling via the radiatively forbidden $3 \mathrm{~s}{ }^{3} \mathrm{~S}^{\mathrm{o}}-3 \mathrm{~s}{ }^{5} \mathrm{~S}^{\mathrm{o}}$ transition. Even though, as in CJ93, we too use the code MULTI, it is a more recent implementation of it (version 2.3) and we have made several other improvements 
in this work. Our atomic model includes a larger number of energy levels. More crucially with respect to the $777 \mathrm{~nm}$ triplet non-LTE corrections, it features updated atomic data, and takes into account background opacities from lines of other elements in the region of the O I UV lines.

Kiselman (1991) studied non-LTE effects on oxygen abundances in solar-type stars, finding that abundance corrections are slightly decreasing from solar to $[\mathrm{Fe} / \mathrm{H}]=-1$ but increasingly large below $[\mathrm{Fe} / \mathrm{H}] \sim-2$, reaching as much as $\sim-0.9$ dex below $[\mathrm{Fe} / \mathrm{H}] \sim-2.5$ (see Fig. 3 in that work). Figure 4 in Kiselman (1991) shows that for a very metal-poor stellar model $([\mathrm{Fe} / \mathrm{H}] \sim-3.5 \mathrm{dex})$ the corrections found were even more severe, with a magnitude of $\left|\Delta \log \epsilon_{\mathrm{O}}\right| \gtrsim 1.4 \mathrm{dex}$. These results, in particular of non-LTE effects which gently decrease at intermediately low metallicity and then steeply increase at very low metallicity, are qualitatively similar to those we obtain when neglecting $\mathrm{H}$ collisions, as Kiselman did. This is fortuitous, due to the opposite effect on the abundance corrections at low $[\mathrm{Fe} / \mathrm{H}]$ of, on one side, our newly adopted electron collision data, and on the other, of both our inclusion of UV background opacity and of the increased completeness of our atomic model ${ }^{11}$. These influences will not matter much for the Sun, where we too find that processes in the line itself are important, due to the line source function drop having a close to two-level character.

Our initial tests with the atomic model by K93 showed that intersystem electron collisions are crucial in the metal-poor regime. The original model in that work gives underestimated abundance corrections due to absence of intersystem coupling. K93 found that the introduction of intersystem collisional coupling changed the equivalent width of the $777 \mathrm{~nm}$ triplet by a few percent and should therefore be of minor importance. We confirm this if testing on atmospheric models with parameters as in that study (for Sun, Procyon and the metal-poor dwarf HD 140283). However, our calculations on atmospheric models with both higher temperature and lower metallicity (typical parameters of very metal-poor turn-off stars) reveal the existence in that range of a larger influence from intersystem coupling on the non-LTE formation of the O I IR triplet. This is due to the fact that in these model atmospheres there is an increased overpopulation of level $3 \mathrm{~s}^{3} \mathrm{~S}^{\mathrm{o}}$, which can easily transmit to the (common) lower level of the triplet lines due to its similar excitation energy.

For the Sun, our investigation obtains non-LTE abundance corrections of $\sim-0.25$ dex for $S_{\mathrm{H}}=0$, in reasonable agreement with the original results of K93. We derive a correction of $\sim-0.15$ dex when including inelastic $\mathrm{H}$ collisions according to the Drawin recipe. Compared to K93 (see also Kiselman 2001), we find that the lower level of the $777 \mathrm{~nm}$ triplet becomes more overpopulated and its upper levels less underpopulated. This effect was already noted via test calculations in Kiselman's work. It is due to atom completeness (i.e. to the combined influence of high-excitation energy levels) caused by the significant effect of recombination cascades due to photon losses in high-lying infrared transitions. Coupling with the large population reservoir in the first ionization stage exists via photon suction through bound-bound transitions. The inclusion of high levels ensures that the consequent efficient downward flow of electrons can be well described. The balance between the contribution of source function drop and line opacity will be better described by our newly employed atomic model. We find an increased contribution of line opacity. Using our atomic model at very low metallicity $([\mathrm{Fe} / \mathrm{H}] \leq-3)$, we find, in particular at higher effective

\footnotetext{
11 Our tests show collisional coupling of high-lying levels to the continuum to be a significant line-weakening channel at low metallicity.
}

temperature, that coupling between high-excitation levels and the continuum works in the opposite sense than in the Sun, causing an upward flow.

Nissen et al. (2002) derived $777 \mathrm{~nm}$ triplet non-LTE corrections for the subset among their sample of metal-poor main sequence and subgiant stars in which those lines had been included in the observational setup. Using a previous version of our same code and the model atom in $\mathrm{K} 93$, and neglecting $\mathrm{H}$ collisions, they found moderate non-LTE corrections. The star with lowest metallicity (and also highest temperature) in their sample is LP815-43, for which they derive the largest non-LTE correction of $\Delta \log \epsilon_{\mathrm{O}}=-0.25 \mathrm{dex}$. When neglecting $\mathrm{H}$ collisions as they do, we obtain $\Delta \log \epsilon_{\mathrm{O}}=-0.55$ dex. Note however that this is likely an overestimate and would bring the non-LTE determination of $[\mathrm{O} / \mathrm{Fe}]$ to very low values. If including $\mathrm{H}$ collisions, the abundance correction becomes $\Delta \log \epsilon_{\mathrm{O}} \sim-0.4$ dex. It is interesting to note that, while their non-LTE corrections show a slight decrease with metallicity down to $[\mathrm{Fe} / \mathrm{H}] \sim-2.5$, there is a hint of a significant increase in the effects at or below the metallicity of LP815-43, as stated at the end of their Sect. 4.3. This is the only star falling in the parameter range where we observe the very large increase in non-LTE corrections. Qualitatively, the trend with metallicity in the non-LTE corrections of Nissen and collaborators reproduces our finding at overlapping metallicities. Since we find collisional coupling not to play a major role until very low metallicity is reached, this explains why only the result for LP815-43 is significantly discrepant. The differences in the atomic models (namely, electron and $\mathrm{H}$ collisions, atom completeness, oscillator strengths) used in the two studies will indeed mostly matter in that regime. The results of Nissen et al. were used by Akerman et al. (2004) by interpolating the non-LTE corrections for relevant parameters, while García Pérez et al. (2006) performed tailored calculations in the same fashion as Nissen et al. The above conclusion - namely, underestimated non-LTE effects at very low metallicity - applies in those cases too. For all stars in the study by García Pérez et al., we obtain (neglecting H collisions as they do) more severe non-LTE abundance corrections, however remaining within $\sim 0.05$ dex of their estimates. This would help bring their derived $[\mathrm{O} / \mathrm{Fe}]$ values from the IR triplet in better agreement with those they determined from [O I], however still without reaching full consistency between the abundance estimates from the two indicators.

Shchukina et al. (2005) neglected H collisions in their study of the impact of non-LTE and granulation effects on Fe and $\mathrm{O}$ in the metal-poor halo subgiant HD 140283. Their resulting 1D non-LTE corrections are smaller $(-0.18 \mathrm{dex})$ than the correction they estimated for the Sun (which is similar to our value for it). Such a trend agrees with our result using an appropriate model atmosphere $\left(T_{\text {eff }}=5690 \mathrm{~K}, \log g=3.67\right.$ and $[\mathrm{Fe} / \mathrm{H}]-2.4)$, for which we too find a smaller abundance correction $(-0.12$ dex $)$ than our estimate for a solar model. Note that HD 140283 lies just outside the (higher effective temperature and lower metallicity) regime where we encounter large non-LTE effects. Moreover, Shchukina et al.'s reanalysis of its stellar parameters, aimed at solving the inconsistencies in the abundance determination, arrive at the conclusion that this star has significantly lower temperature and higher metallicity than previously thought.

Finally, regarding comparison with the work of Meléndez et al. (2006), they computed non-LTE corrections with the NATAJA code (see Shchukina \& Trujillo Bueno 2001), finding a roughly constant oxygen-to-iron overabundance (mean $[\mathrm{O} / \mathrm{Fe}] \sim+0.5)$ from published data on unevolved metal-poor halo stars. Note that their Fig. 4 shows that the lowest-metallicity 


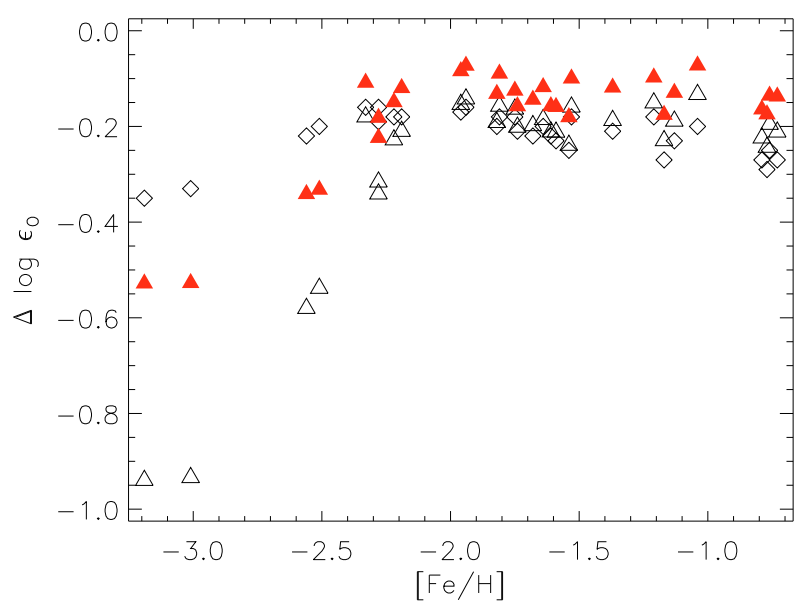

Fig. 9. Non-LTE abundance corrections versus metallicity, for the O I IR $777 \mathrm{~nm}$ triplet. Open diamonds indicate the results of Meléndez et al. (2006), while the other symbols mark our corrections for $S_{\mathrm{H}}=0$ and $S_{\mathrm{H}}=1$ (open and filled triangles respectively).

stars tend to have smaller $[\mathrm{O} / \mathrm{Fe}]$ values than what is found around $[\mathrm{Fe} / \mathrm{H}] \sim-2$. This may indicate (see Nissen et al. 2007) too high temperatures at low $[\mathrm{Fe} / \mathrm{H}]$, a possibility either related to uncertainties in the reddening or to the not-yet settled $T_{\text {eff }}{ }^{-}$ scale itself. Collisions with $\mathrm{H}$ were not included in their work (Meléndez, private communication). A comparison of the nonLTE corrections shows that theirs are significantly smaller at low $[\mathrm{Fe} / \mathrm{H}]$. Inspecting their Fig. 5, where they compare with the non-LTE corrections of Akerman et al. (2004), the star with the largest non-LTE effect $\left(\Delta \log \epsilon_{\mathrm{O}} \sim-0.35 \mathrm{dex}\right)$ is G64-12, which has the lowest metallicity in their literature sample. For a model with similar parameters as those used by them, we obtain a larger abundance correction $(\sim-0.5 \mathrm{dex}$, or more if neglecting $\mathrm{H}$ collisions as they did). As seen in Fig. 9, while also the non-LTE corrections of Meléndez and collaborators tend to become more significant at $[\mathrm{Fe} / \mathrm{H}]<-2$, we find that the trend with decreasing metallicity is much more pronounced, with more significant non-LTE abundance corrections than found by those authors, thus likely destroying their claimed quasi-flat $[\mathrm{O} / \mathrm{Fe}]$ trend with metallicity, even when adopting the results we obtained in the case of efficient $\mathrm{H}$ collisions. The differences are likely related to the use of different codes, model atmospheres, opacities, and to our inclusion in the oxygen model atom of new electronimpact data.

\subsection{Gratton et al. (1999)}

Using an earlier version of the same code employed here and a similar model atom to the one in K93, but Kurucz (1992) model atmospheres, Gratton et al. (1999) studied departures from LTE in F-K stars for a broad range of gravities and metallicities, and including collision with electrons and hydrogen. For the latter process, they adopted very high efficiency (scaling factor $S_{\mathrm{H}} \sim 3.2$ in our notation), based on an empirical calibration on RR Lyrae stars, i.e. on the requirement that the $777 \mathrm{~nm}$ and $616 \mathrm{~nm}$ triplets should provide the same abundances. Forcing such agreement may however mean that other assumptions are folded into (and remain hidden in) the scaling parameter for $\mathrm{H}$ collisions. Based on previous evidence from solar investigations (Allende Prieto et al. 2004), there is some indication that the use of Drawin's recipe with no scaling factor (i.e., $S_{\mathrm{H}}=1$ ) may be reasonable for the O I IR triplet. Since collisions with neutral $\mathrm{H}$ atoms are generally considered the most important thermalizing process in late-type stars, Gratton et al.'s higher choice for $S_{\mathrm{H}}$ will give smaller non-LTE corrections than in our case. Indeed, those authors found relatively mild non-LTE effects on the OI $777 \mathrm{~nm}$ triplet, in particular $\sim-0.1$ dex in the Sun. Their study reaches down to $[\mathrm{Fe} / \mathrm{H}]=-3$, where for hot, high-gravity atmospheric models they find the largest corrections (reaching $\Delta \log \epsilon_{\mathrm{O}} \sim-0.5 \mathrm{dex}$ ) for the O I IR triplet. Choosing atmospheric parameters in common between the two studies $\left(T_{\text {eff }}=6000 \mathrm{~K}, \log g=4.5,[\mathrm{Fe} / \mathrm{H}]=-3\right.$, see their Fig. 12), and very efficient $H$ collisions as they did, we too obtain small non-LTE abundance corrections (only -0.07 dex in our case, i.e. even a few hundredths of a dex less severe than in Gratton et al.). However, if adopting $S_{\mathrm{H}} \leq 1$, our nonLTE corrections will actually be larger $\left(\Delta \log \epsilon_{\mathrm{O}} \lesssim-0.25 \mathrm{dex}\right)$. Differences with our study are therefore mostly due to our use of $\mathrm{H}$ collisions more in line with the majority of presently available evidence, and only to a lesser extent to employing different model atmospheres. It is interesting to note (their Fig. 12) that their non-LTE corrections for the hottest model used by them $\left(T_{\text {eff }}=7000 \mathrm{~K}\right)$ show a significant increase when going from $[\mathrm{Fe} / \mathrm{H}]=-2$ to -3 . The results of Gratton and co-workers were for example included in Carretta et al. (2000) and in Primas et al. (2001), deriving a "quasi-flat" oxygen-to-iron overabundance with mean $[\mathrm{O} / \mathrm{Fe}] \sim+0.5$ at low metallicity.

\subsection{Takeda (1992, 1994, 2003); Takeda \& Honda (2005)}

Takeda published a series of non-LTE studies of oxygen. In his investigations, rates due to collisions with $\mathrm{H}$ atoms were in general computed using the Drawin recipe without corrections.

Takeda (1994) found $\left|\Delta \log \epsilon_{\mathrm{O}}\right|<0.1$ dex for the Sun but $\sim-0.4$ dex for a hotter model representative of Procyon (for which, when including $\mathrm{H}$ collisions, we derive a correction of $-0.4 /-0.5$ dex depending on the OI triplet line considered). That author also attributed to non-LTE effects, at least qualitatively, the discrepancy between oxygen abundances from the triplet and forbidden lines generally found in studies of low- $[\mathrm{Fe} / \mathrm{H}]$ halo stars. In Takeda's non-LTE analysis, the most oxygen-deficient $(\log \mathrm{O}=6.86)$ calculation starts to show the signs of UV pumping in the resonance lines (see their Fig. 9 and discussion in the corresponding section).

Takeda (2003) gives an analytical formula, independent of metallicity, to approximate the non-LTE abundance corrections resulting from his calculations, arguing that it will provide a fairly good approximation for lines with $W_{\lambda}<100 \mathrm{~m} \AA$. For the Sun (see also Takeda \& Honda 2005) and for solar-type dwarfs at moderately low $[\mathrm{Fe} / \mathrm{H}]$, our calculations including $\mathrm{H}$ collisions give similar results to those of Takeda. The mentioned formula by Takeda does not explicitly contain $[\mathrm{Fe} / \mathrm{H}]$. However, given its dependence on line strength, it will give generally less severe abundance corrections at low metallicity due to weaker lines, so that our non-LTE corrections become larger than Takeda's at low metallicity. Note that his Fig. 4 shows that estimated corrections are low and roughly constant $(\sim-0.1 \mathrm{dex})$ for the stars considered in his comparison with Nissen et al. (2002). As discussed in Sect. 4.1, we obtain roughly similar non-LTE corrections to Nissen et al. (2002), or even more severe effects for the lowestmetallicity star in their sample. Note however that when adopting efficient $\mathrm{H}$ collisions as in the study by Takeda, the results will be closer. The absence of a significant trend of the nonLTE corrections with decreasing metallicity found by Takeda (2003) is at variance with the conclusions of e.g. Carretta et al. (2000). Takeda argues that, even though in shallow atmospheric 
layers there is an appreciable increase of the non-LTE line opacity at low metallicity, this does not affect the deeply-forming $777 \mathrm{~nm}$ O I triplet, so that fine details of the atomic model will not matter, due to a close-to-two-level line source function. Note that Takeda does in fact find a metallicity dependence when neglecting $\mathrm{H}$ collisions. We find that at low metallicity, the twolevel nature of the non-LTE effect breaks down. In particular, for $T_{\text {eff }}=6500 \mathrm{~K}, \log g=4$, using Takeda's formula with our estimated $W_{\text {non-LTE }}$ would give a constant $\Delta \log \epsilon_{\mathrm{O}} \sim-0.1 \mathrm{dex}$ at very low metallicity, compared to our results of $\sim-0.5$ dex and $\sim-0.9$ dex (respectively for $[\mathrm{Fe} / \mathrm{H}]=-3$ and -3.5 ) when adopting $\mathrm{H}$ collisions. In this respect, our abundance corrections would be more helpful in solving the oxygen discrepancy, bringing the abundances derived from the $777 \mathrm{~nm}$ triplet in better agreement with the usually lower value from [O I]-based LTE estimates.

Thus, we do not confirm the small dependence of O I nonLTE corrections from $[\mathrm{Fe} / \mathrm{H}]$ found by Takeda. That author (Takeda, private communication) included intersystem collisional coupling using Auer \& Mihalas (1973) and, in Takeda (1992) and successive works, treatment of scattering in the continuum source function. Note that Takeda accounted for background line opacity in the UV only from $\mathrm{H}$ lines. However, as seen from our tests, metal lines tend to make the non-LTE effect smaller. Therefore, this actually makes the discrepancy with our results more significant, and likely explainable due to the summed effect of different atomic data, and use of different model atmospheres. The results in Takeda (2003) were applied by means of interpolation/extrapolation in Takeda \& Honda (2005), giving a linear increase of $[\mathrm{O} / \mathrm{Fe}]$ with $[\mathrm{Fe} / \mathrm{H}]$.

\subsection{Ramírez et al. (2007)}

In their study of a large sample of nearby stars, Ramírez et al. (2007) have derived non-LTE corrections (neglecting the role of inelastic $\mathrm{H}$ collisions) for a grid of model atmospheres, mainly covering the metallicity regime of the galactic thin and thick disks. Their adopted O I model atom is explained in detail in Allende Prieto et al. (2003a,b). It consists of 54 levels plus continuum and 242 transitions, with the radiative data coming from the Opacity Project and NIST, and electron collision rates estimated via approximate formulae. For excitation by electrons in forbidden transitions, they computed the collision rate coefficient from Eissner \& Seaton (1974), adopting an arbitrary choice of effective collision strength. As shown in the detailed comparison by Barklem (2007a), the data employed by us for radiatively forbidden transitions show poor agreement with those estimated by Allende Prieto et al. In particular, for the radiatively forbidden transition which we find important in coupling triplet and quintet systems, our adopted rate is two orders of magnitude larger.

The non-LTE corrections of Ramírez et al. reach down to $\sim-0.4$ dex at $[\mathrm{Fe} / \mathrm{H}] \sim-1.5$ (their Fig. 7). For stars with determined metallicities, as from their Table 6, the largest corrections are $\sim-0.3$ dex (e.g., HD 112887 at $T_{\text {eff }}=6319 \mathrm{~K}, \log g=3.88$, $[\mathrm{Fe} / \mathrm{H}]=-0.38)$. Their abundance corrections show a relatively mild dependence on metallicity for the range studied. Since our investigation extends to much lower metallicity than theirs, we only compared the results for a few representative stars with atmospheric parameters covered by both works, using the routine (Ramírez, private communication) mentioned in their paper. The star with lowest determined metallicity in Table 6 of Ramírez et al. (2007) has $[\mathrm{Fe} / \mathrm{H}]=-1.46$. For this star (HD 132475), their routine gives a non-LTE correction of $\sim-0.28$ dex with their adopted parameters of $T_{\text {eff }}=5613 \mathrm{~K}$ and $\log g=3.81$. Our nonLTE correction for similar parameters is smaller, $\sim-0.17 \mathrm{dex}$.

For a standard star like Procyon $\left(T_{\mathrm{eff}}=6530 \mathrm{~K}, \log g=3.96\right.$, $[\mathrm{Fe} / \mathrm{H}]=0$ ), for which their formula would give $\sim-0.28 \mathrm{dex}$, we derive a much larger estimate of $\sim-0.55 \mathrm{dex}$, when adopting $S_{\mathrm{H}}=0$. Note however that, as discussed, a choice of $S_{\mathrm{H}} \sim 1$ may be more appropriate, which would lead to a value of $\Delta \log \epsilon_{\mathrm{O}} \sim$ -0.45 dex in our case.

For the Sun, their non-LTE correction is roughly $\sim-0.15$ dex, which is $\sim 0.1$ dex less severe than in our case. Even adopting their slightly higher (LTE) solar oxygen abundance, our results for the Sun would not change noticeably. A choice of $S_{\mathrm{H}} \sim 1$ for $\mathrm{H}$ collisions in our case would bring, however fortuitously, our estimate close to that of Ramírez et al.

\section{Implications}

Our results have important consequences for the debate regarding the $[\mathrm{O} / \mathrm{Fe}]$ trend at low metallicity, and on the understanding of the chemical evolution of this element in the early Galaxy. The large difference often found in the literature between the oxygen content of old stars as derived via the O I IR triplet and the other oxygen abundance indicators (in particular, [O I]) is likely to be explained in terms of various uncertainties acting to produce disagreeing estimates (Asplund 2005; Meléndez et al. 2006).

In our re-assessment of non-LTE effects on the O I IR triplet, we have found that at low metallicity they are more important than what is often assumed in the literature. In Fig. 10, we show the effect of applying our abundance corrections to literature data by Israelian et al. (2001), Nissen et al. (2002) and Meléndez et al. (2006). Since uncertainties in H collision efficiency affect the modeling of the $777 \mathrm{~nm}$ O I triplet (and corresponding non-LTE corrections) very significantly, we have plotted results derived applying $S_{\mathrm{H}}=0$ and $S_{\mathrm{H}}=1$, respectively. The limited observational evidence for the Sun suggests for oxygen rather efficient thermalization via impacts with $\mathrm{H}$ atoms, akin to choosing Drawin's formula without any scaling factor $\left(S_{\mathrm{H}}=1\right)$. The severe non-LTE corrections we find seem to largely erase the linear increasing trend towards low metallicity sometimes found in LTE. Our estimates with a choice of $S_{\mathrm{H}}=1$ suggest a rather flat trend below $[\mathrm{Fe} / \mathrm{H}]=-1$, although with significant residual scatter. Only adopting an unrealistically high amount of thermalization would make the non-LTE effects negligible. When instead completely ignoring $\mathrm{H}$ collisions, (as discussed, however, this case may be unrealistic), the $[\mathrm{O} / \mathrm{Fe}]$ values in Fig. 10 would become even lower (the trend turning into a decrease with decreasing metallicity). If such a model was borne out, it would possibly require new investigations on the efficiency of the ${ }^{12} \mathrm{C}(\alpha, \gamma){ }^{16} \mathrm{O}$ nuclear reaction and on published nucleosynthetic yields at low metallicity.

We note that our non-LTE corrections do not destroy the good agreement between [OI] and OI abundances found by Nissen and collaborators in five of their sample stars. Since those stars are between $-2.42<[\mathrm{Fe} / \mathrm{H}]<-1.04$ they do not suffer the very large corrections (nor the large sensitivity to $\mathrm{H}$ collisions) we found at very low metallicity. In fact, our resulting non-LTE corrections, and thus estimated abundances from the $777 \mathrm{~nm}$ triplet, agree with those of Nissen et al. (2002) within 0.1 dex for those stars.

Our results may imply that the oxygen-to-iron overabundance does not depend significantly on metallicity down to $[\mathrm{Fe} / \mathrm{H}]=-3$, with an essentially flat trend (this may include a slight slope in either direction) at very low metallicity. This is in line with the behaviour recently found via accurate 

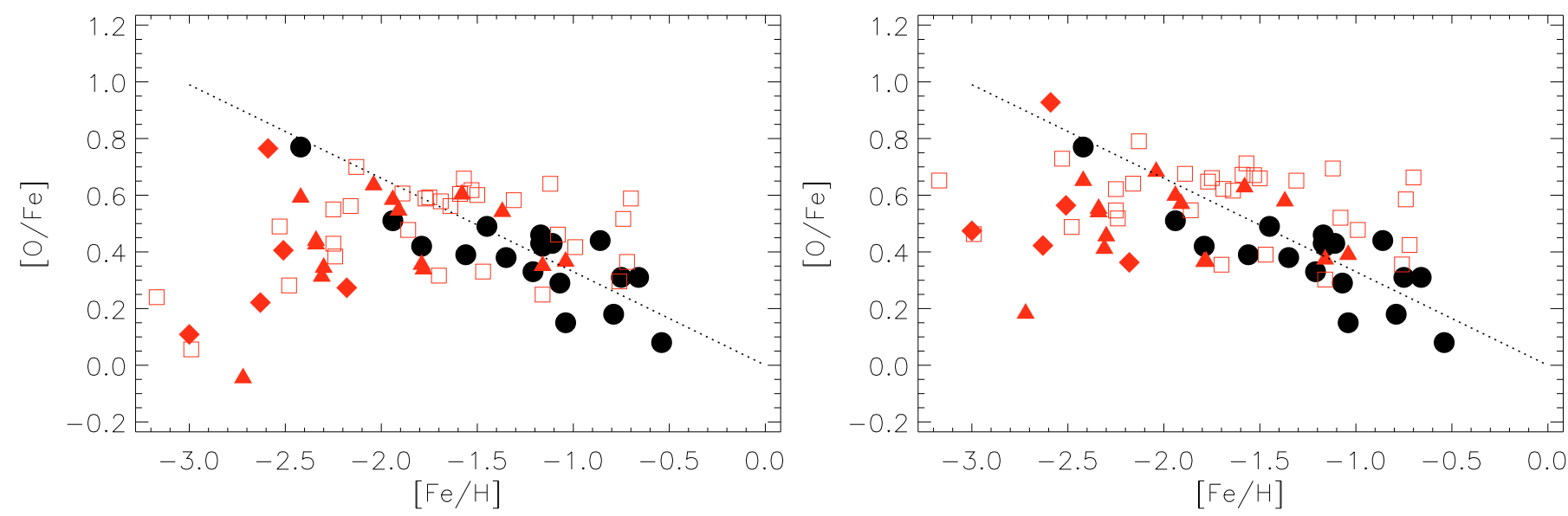

Fig. 10. Revised $[\mathrm{O} / \mathrm{Fe}]$ versus $[\mathrm{Fe} / \mathrm{H}]$, from literature data. The different symbols are: filled circles, for [OI]-based, LTE-obeying values from Nissen et al. (2002); filled triangles, for the 1D estimates (from that same study, but non-LTE corrected according to our results) based on the O I $777 \mathrm{~nm}$ triplet; filled diamonds for O I-based values for the stars in Israelian et al. (2001) with fully determined stellar parameters and oxygen triplet abundances in their study, taking into account our non-LTE corrections on the oxygen triplet; and empty squares, for our non-LTE corrected estimates using the Meléndez et al. (2006) data set (after adjusting $T_{\text {eff }}$-scale, see Nissen et al. 2007). The non-LTE abundance corrections we applied were computed with a choice of $S_{\mathrm{H}}=0$ and $S_{\mathrm{H}}=1$ (left and right panel respectively) for hydrogen collisions. The dotted line indicates the mean trend suggested by Israelian et al. (2001), i.e. $[\mathrm{O} / \mathrm{Fe}]=-0.33[\mathrm{Fe} / \mathrm{H}]$.

abundance estimates (taking into account non-LTE effects) for other typical $\alpha$-capture elements (e.g. Nissen et al. 2007). In Fabbian et al. (2009), we further investigate this issue, together with a study of the $[\mathrm{C} / \mathrm{Fe}]$ and $[\mathrm{C} / \mathrm{O}]$ ratios at low metallicity, using new observational data.

We have shown that O I LTE abundances derived from the $777 \mathrm{~nm}$ triplet are likely large overestimates, especially at low $[\mathrm{Fe} / \mathrm{H}]$, but there is still significant uncertainty related to the choice of $\mathrm{H}$ collision efficiency. Some authors have argued on astrophysical grounds that assuming the Drawin estimate might be reasonable, at least for the Sun. Solar center-to-limb variation in fact not only provides evidence that the O I $777 \mathrm{~nm}$ lines are formed in non-LTE (Altrock 1968; Shchukina 1987; Kiselman 1991), but also suggests a possible significant role of $\mathrm{H}$ collisions (e.g. Allende Prieto et al. 2004). Relative to electron collisions, these should be even more important at low metallicity. The model with $S_{\mathrm{H}}=1$ seems to predict lines in better agreement with astrophysical expectations from other oxygen spectral features. However, this is only an indication, since it could well result from errors due to the Drawin estimates and to the use of 1D atmospheric models coincidentally acting in opposite directions. Here, we too find that $\mathrm{H}$ collisions may need to be taken into account for this element, by reasoning on the otherwise unlikely large impact of non-LTE corrections on $[\mathrm{O} / \mathrm{Fe}]$ at very low metallicity, when they are neglected. At the same time, we warn that in fact (however unavoidable in the absence of available detailed quantum mechanical computation for relevant $\mathrm{O}+\mathrm{H}$ collisions, which are urgently needed together with constraints via solar observations), adopting the general Drawin recipe - possibly with some scaling factor - for all transitions is a rough approximation, since different transitions likely have different sensitivity to such collisions.

Realistic cross-sections for continuum absorption are also needed. We have found that $\mathrm{Si}$ abundance plays an important role (via bound-free absorption) in the formation of the IR oxygen triplet at very low metallicity. This element is commonly thought to follow the normal behaviour of other $\alpha$-elements in being overabundant compared to $\mathrm{Fe}$ at low metallicity. Cayrel et al. (2004) recently obtained an average $[\mathrm{Si} / \mathrm{Fe}] \sim+0.4$ (with small scatter), which we have adopted in the calculations. However, very recently Nissen $\&$ Schuster (2008) have found evidence that halo stars may fall into two groups, with distinct $\alpha$-enhancement. If this significant $(\sim 0.20$ dex $)$ separation between "low- $\alpha$ " and "high- $\alpha$ " halo stars is preserved down to very low $[\mathrm{Fe} / \mathrm{H}]$ for silicon, it may well be an important factor in the discussion of the oxygen problem, because a lower content of Si would imply larger non-LTE effects on the O I triplet, for an otherwise fixed set of atmospheric parameters. Any residual star-to-star differences in the Si content of very metal-poor halo stars may thus play some role in the scatter observed in $[\mathrm{O} / \mathrm{Fe}]$ versus $[\mathrm{Fe} / \mathrm{H}]$. It is therefore important, in future studies on the oxygen problem, to pin down the non-LTE corrections by also having very accurate determinations of the stellar silicon content.

We cannot yet completely rule out the existence of a continuous steeply increasing trend of $[\mathrm{O} / \mathrm{Fe}]$ with decreasing $[\mathrm{Fe} / \mathrm{H}]$. However, its existence would require extremely efficient $\mathrm{H}$ collisions (e.g. $S_{\mathrm{H}} \gtrsim 3$ ), in order to bring the abundance estimates close to the high extreme set by the LTE expectation.

The oxygen-to-iron overabundance more likely reaches $\lesssim+0.5$ dex at very low metallicity, with a plateau or slight metallicity-dependence. Further modeling and new high-quality observations will be needed to completely settle the issue, but models of galactic chemical evolution constrained by the $[\mathrm{O} / \mathrm{Fe}]$ ratio derived from observations of stars at low metallicity can now hope to reach a more definite conclusion on possible scenarios required by derived stellar composition at early stages. Such low (compared to LTE) $[\mathrm{O} / \mathrm{Fe}]$ values in galactic halo stars are in better agreement with recent estimates (Péquignot 2008) for low-metallicity blue compact dwarf galaxies.

Regarding the solar oxygen abundance, it is little affected by the new electron collisions. The determination of Asplund et al. (2004) employed several different indicators obtaining remarkable agreement between them, by performing a 3D nonLTE analysis. Our 1D non-LTE results for the $777 \mathrm{~nm}$ IR triplet lines agree within $\sim 0.05$ dex with those published by Asplund et al., when adopting their choice of $S_{\mathrm{H}}=0$. We argue that, even if $\mathrm{H}$ collisions were important for oxygen - in which case our estimates would give up to $\sim 0.1$ dex higher oxygen abundance for some lines (in particular, for the $844.67 \mathrm{~nm}$ line) due to smaller corrections than theirs - the overall change when 
averaging among all lines will remain within 0.05 dex. This is still significant, since the derived solar oxygen abundance may be around $\sim 8.70$, which would partially alleviate the current discrepancy with helioseismology results.

Incidentally, we cannot explain the extremely large $[\mathrm{O} / \mathrm{Fe}]$ overabundances derived in some cool giants at very low metallicity, and the very different abundances derived in those objects from permitted and forbidden oxygen lines respectively (Israelian et al. 2004), because in that range our non-LTE corrections tend to become $\sim-0.1$ dex or less severe. To gain a better insight into the various outstanding problems, detailed results from 3D non-LTE investigations will be necessary.

In a series of papers, Schuler et al. (2004, 2005, 2006) have studied solar-type open cluster dwarfs, finding much lower mean [O I]-based abundances than for results based on the permitted O I triplet. From the latter, they found a puzzling and abrupt increase in LTE oxygen abundance derived from the highexcitation triplet for stars with $T_{\text {eff }} \lesssim 5800 \mathrm{~K}$. We exclude that this trend could be due for the most part to departures from LTE, since our resulting abundance corrections tend to be relatively small in the relevant temperature range.

\section{Conclusions}

We have explored the oxygen non-LTE line formation over a wide parameter space. Thanks to several improvements mainly, to detailed treatment of UV radiation field including opacities by background lines, and extended modeling including recently available atomic data - we derive estimated corrections at low $[\mathrm{Fe} / \mathrm{H}]$ to LTE abundances derived from the IR $777 \mathrm{~nm}$ triplet that are generally larger than usually adopted in the literature. It is clear that the availability of adequate atomic data is crucial to achieve a reliable non-LTE solution for this and other important astrophysical problems. Using rates derived from newly available estimates for electron-impact excitation by electrons (Barklem 2007a), we have performed non-LTE calculations in one-dimensional stellar atmospheric models of late-type stars extending to very low metallicity, in order to understand the formation of the permitted neutral atomic oxygen lines. The trends of non-LTE corrections across the parameter space can be understood in terms of the different non-LTE mechanisms in action: mainly source function drop at solar metallicity, and level overpopulation at low $[\mathrm{Fe} / \mathrm{H}]$.

For solar-type dwarfs, the non-LTE corrections we found show relatively little metallicity-dependence down to $[\mathrm{Fe} / \mathrm{H}] \sim$ -2 . This is in line with previous findings by other authors (e.g., Takeda 2003). However, at the higher temperatures of turn-off stars at low $[\mathrm{Fe} / \mathrm{H}]$, the departures from LTE tend to increase to large values when metallicity decreases, so that LTE abundances will require increasingly large corrections. The two-level approximation valid for the Sun breaks down and results no longer mostly depend on processes in the line itself. The large non-LTE corrections at low $[\mathrm{Fe} / \mathrm{H}]$ follow from increased collisional intersystem coupling. The (over)population of the lower level of the $777 \mathrm{~nm}$ triplet tends to increase and so does line opacity. The formation of the $777 \mathrm{~nm}$ lines becomes sensitive, at low metallicity, to absorption processes in the UV continuum. This makes the non-LTE corrections metallicity-dependent due to the influence of $\mathrm{Si}$ absorption. The results have an impact on the derivation of the "true" $[\mathrm{O} / \mathrm{Fe}]$ trend at low metallicity, with a roughly constant plateau from literature data if including thermalization by collisions with hydrogen atoms.
The problem of $\mathrm{H}$ collisions is obviously still very much open. Progress is required, via theoretical quantum mechanical calculations or via limb darkening observations (Allende Prieto et al. 2004), in order to assess whether $H$ collisions can provide efficient thermalization. This is of high priority also for a more conclusive determination of the solar oxygen content as the standard of reference in $\mathrm{O}$ abundance determinations. Some authors (e.g., Kiselman 1991; Nissen et al. 2002; Asplund et al. 2004) have preferred to completely neglect $\mathrm{H}$ collisions, because of experimental (e.g. Fleck et al. 1991) and theoretical (e.g. Barklem et al. 2003) evidence that Drawin's formula gives usually too large (by orders of magnitude) estimates for atmospheres of cool stars, at least for simple atoms like Li. We find that even with a choice of $S_{\mathrm{H}}=1$, the non-LTE corrections would still remain large at very low metallicity (up to $-0.85 \mathrm{dex}$ at $[\mathrm{Fe} / \mathrm{H}]=-3.5$ ).

Even though when adopting extreme choices of the parameter regulating the efficiency of $\mathrm{H}$ collisions, the difference in the resulting $777 \mathrm{~nm}$ triplet non-LTE corrections is relatively small towards higher metallicity, it remains significant and $\sim 0.1 \mathrm{dex}$ for the Sun, in the sense of efficient collisions producing smaller non-LTE effects (which would help to partially alleviate the discrepancy with helioseismology, at least for oxygen).

It looks as though a combination of $3 \mathrm{D} /$ non-LTE effects, choice of model atmospheres and of temperature scale, and observational uncertainties both in derived stellar parameters and equivalent width measurements have conspired to prevent a clear solution of the oxygen problem so far. It now seems as though better agreement with the abundance from the forbidden [O I] lines (which are unaffected by non-LTE effects) and from $\mathrm{OH}$ molecular lines (once they are corrected for 3D effects, Asplund \& García Pérez 2001) can be obtained when non-LTE effects are taken into account for the $777 \mathrm{~nm}$ oxygen triplet abundance, see e.g. Meléndez et al. (2006). Our results seem to suggest that large non-LTE corrections at low metallicity are the norm, at least for turn-off halo stars.

Given our findings concerning the importance of the radiatively forbidden $3 s^{3} S^{0}-3 s{ }^{5} S^{0}$ transition, it is crucial to check if a similar behaviour is seen for other atoms with similar structure, e.g. C, N, and S, or even in all atoms.

Non-LTE corrections obtained with ATLAS models turned out to be very similar, for a range of atmospheric parameters, to those we found using MARCS models. However, at very low metallicity, significant discrepancies appear, with large differences between non-LTE corrections using the two sets of models. Interestingly, this metallicity range overlaps with that for which the debate between "flat" and "linear" $[\mathrm{O} / \mathrm{Fe}]$ trend exists. However, as seen from Fig. 10, our main conclusions will not change even if applying ATLAS non-LTE corrections, which would give, around $[\mathrm{Fe} / \mathrm{H}]=-2.5$, smaller corrections by $\sim 0.3$ dex in the left panel (no $\mathrm{H}$ collisions) and by $\sim 0.2$ dex in the right panel (H collisions "a la Drawin"). Anyhow, more sophisticated modeling of stellar atmospheres is likely another crucial factor with respect to the oxygen problem, in particular for abundances from $\mathrm{OH}$ lines, but also to understand how the large non-LTE effects we found act in a 3D atmosphere. Therefore, it is obviously urgent to carry out, for a large range of stellar parameters, full 3D non-LTE calculations, in order to further investigate the formation of the oxygen lines at low $[\mathrm{Fe} / \mathrm{H}]$. It is expected that non-LTE effects will be enhanced when using 3D atmospheric models, due to their cooler superficial temperatures, to which the high-excitation lines of interest are known to be sensitive. This crucial step forward will allow enhanced abundance analyses and will help to shed light on early galactic chemical evolution, contributions by SN II and massive stars, time delay 
of yields from type Ia SNe, and the reality of the $[\mathrm{C} / \mathrm{O}]$ upturn at low metallicity (Akerman et al. 2004; Spite et al. 2005; Fabbian et al. 2009). It is likely that - together with the use of an accurate temperature scale - when finally taking into account, using detailed calculations with the best available atomic data, the interplay of 3D and non-LTE effects in the formation of the various available abundance indicators (including for the determination of metallicity), it will be possible to in the end fully solve the "oxygen problem".

Here, we have demonstrated that non-LTE corrections play an important role towards a solution. They will need to be taken into consideration in order to abate large systematic errors still afflicting LTE-based estimates. To check whether agreement between abundances from the different oxygen lines can be found at low metallicity will furthermore require using improved observational data, in particular from a large sample of subgiants or giants where more oxygen indicators are available. This important test will help to clarify outstanding issues related to the $[\mathrm{O} / \mathrm{Fe}]$ controversy.

Acknowledgements. This work has been partly funded by the Australian Research Council (grants DP0342613 and DP0558836). D.F. is grateful to the Department of Astronomy and Space Physics, Uppsala Astronomical Observatory, Uppsala, Sweden, for its hospitality. D.F. would also like to thank Remo Collet, Jorge Meléndez and Poul Erik Nissen for fruitful discussion and comments. P.B. is a Royal Swedish Academy of Sciences Research Fellow supported by a grant from the Knut and Alice Wallenberg Foundation. P.B. also acknowledges the support of the Swedish Research Council. This research has made use of NASA's Astrophysics Data System, of the NIST Atomic Spectra Database (version 3), which is operated by the National Institute of Standards and Technology, and of the Vienna Atomic Line Database. We are indebted to Iván Ramírez for providing the routine which we used to compare with his nonLTE oxygen results, and to Yoichi Takeda, for details on his non-LTE work.

\section{References}

Abia, C., \& Rebolo, R. 1989, ApJ, 347, 186

Abrahamsson, E., Krems, R. V., \& Dalgarno, A. 2007, ApJ, 654, 1171

Akerman, C. J., Carigi, L., Nissen, P. E., Pettini, M., \& Asplund, M. 2004, A\&A, 414,931

Allende Prieto, C., Lambert, D. L., Hubeny, I., \& Lanz, T. 2003a, ApJS, 147, 363

Allende Prieto, C., Hubeny, I., \& Lambert, D. L. 2003b, ApJ, 591, 1192

Allende Prieto, C., Asplund, M., \& Fabiani Bendicho, P. 2004, A\&A, 423, 1109

Altrock, R. C. 1968, Sol. Phys., 5, 260

Arnett, W. D. 1978, ApJ, 219, 1008

Asplund, M. 2004, A\&A 417, 769

Asplund, M. 2005, ARA\&A, 43, 481

Asplund, M., \& García Pérez A. E. 2001, A\&A, 372, 601

Asplund, M., Gustafsson, B., Kiselman, D., \& Eriksson, K. 1997, A\&A, 318, 521

Asplund, M., Grevesse, N., Sauval, A. J., Allende Prieto, C., \& Kiselman, D. 2004, A\&A, 417, 751

Auer, L. H., \& Mihalas, D. 1973, ApJ, 184, 151

Balachandran, S. C., \& Bell, R. A. 1998, Nature, 392, 791

Balachandran, S. C., Carr, J. S., \& Carney, B. W. 2001, New Astron. Rev., 45, 529

Barbuy, B. 1988, A\&A, 191, 121

Barbuy, B., Meléndez, J., Spite, M., et al. 2003, AJ, 588, 1072

Barklem, P. S. 2007a, A\&A, 462, 781

Barklem, P. S. 2007b, A\&A, 466, 327

Barklem, P. S., Belyaev, A. K., \& Asplund, M. 2003, A\&A, 409, L1

Baschek, B., Scholz, M., \& Sedlmayr, E., 1977, A\&A, 55, 375

Belyaev, A. K., Grosser, J., Hahne, J., \& Menzel, T. 1999, Phys. Rev. A, 60, 2151

Belyaev, A. K., \& Barklem, P. S. 2003, Phys. Rev. A, 68, 62703

Bessell, M. S., Sutherland, R. S., \& Ruan, K. 1991, ApJ, 383, L71

Boesgaard, A. M., King, J. R., Deliyannis, C. P., \& Vogt, S. S. 1999, AJ, 117 492
Carlsson, M. 1986, Uppsala Astronomical Observatory Report, 33

Carlsson, M., \& Judge, P. G. 1993, ApJ, 402, 344 (CJ93)

Carretta, E., Gratton R. G., \& Sneden, C. 2000, A\&A, 356, 238

Castelli, F., \& Kurucz, R. L. 2004, in Modelling of Stellar Atmospheres, ed. N. Piskunov et al., Proc. IAU Symp., 210

Cayrel, R., Depagne, E., Spite, M., et al. 2004, A\&A, 416, 1117

Clayton, D. 2003, Handbook of Isotopes in the Cosmos (Cambridge University Press)

Clementini G., Carretta E., Gratton R. G., et al. 1995, AJ, 110, 2319

Conti, P. S., Greenstein, J. L., Spinrad, H., Wallerstein, G., \& Vardya, M. S. 1967, ApJ, 148, 105

Depagne, E., Hill, V., Spite, M., et al. 2002, A\&A, 390, 187

Drawin, H. W. 1968, Z. Phys., 211, 404

Drawin, H. W. 1969, Z. Phys., 225, 483

Fabbian, D., Asplund, M., Carlsson, M., \& Kiselman, D. 2006, A\&A, 458, 899

Fabbian, D., Nissen, P. E., Asplund, M., Pettini, M., \& Akerman, C. 2009, A\&A, 500,1143

Fleck, I., Grosser, J., Schnecke, A., Steen, W., \& Voigt, J. 1991, J. Phys. B, 24, 4017

Fulbright, J. P., \& Johnson, J. A. 2003, ApJ, 595, 1154

García Pérez A. E., Asplund, M., Primas, F., Nissen, P. E., \& Gustafsson, B. 2006, A\&A, 451, 621

Gratton, R. G., \& Ortolani, S. 1986, A\&A, 169, 201

Gratton, R. G., Carretta, E., Eriksson, K., \& Gustafsson, B. 1999, A\&A, 350, 955

Gustafsson, B., Bell, R. A., Eriksson, K., \& Nordlund, Å. 1975, A\&A, 42, 407

Gustafsson, B., Edvardsson, B., Eriksson, K., et al. 2008, A\&A, 486, 951

Israelian, G., García López, R. J., \& Rebolo, R. 1998, ApJ, 507, 805

Israelian, G., Rebolo, R., García López, R. J., et al. 2001, ApJ, 551, 833

Israelian, G., Shchukina, N., Rebolo, R. et al. 2004, A\&A, 419, 1095

Johnson, H. R., Milkey, R. W., \& Ramsey, L. W. 1974, ApJ, 187, 147

King, J. R. 1993, AJ, 106, 1206

Kiselman, D. 1991, A\&A, 245, L9

Kiselman, D. 1993, A\&A, 275, 269 (K93)

Kiselman, D. 2001, New Astron. Rev., 45, 559

Kraft, R. P., Sneden, C., Langer, G. E., \& Prosser, C. F. 1992, AJ, 104, 645

Krems, R. V., Jamieson, M. J., \& Dalgarno, A. 2006, ApJ, 647, 1531

Kurucz R. L. 1992, Rev. Mex. Astron. Astrofis., 23, 181

Matteucci, F., \& Greggio, L. 1986, A\&A, 154, 279

Matteucci, F., \& François, P. 1992, A\&A, 262, L1

Meléndez, J., \& Barbuy, B. 2002, AJ, 575,474

Meléndez, J., Shchukina, N. G., Vasiljeva, I. E., \& Ramírez, I. 2006, ApJ, 642, 1082

Mishenina, T. V., Korotin, S. A., Klochkova, V. G., \& Panchuk, V. E. 2000, A\&A, 353,978

Nissen, P. E., Primas, F., Asplund, M., \& Lambert, D. L. 2002, A\&A, 390, 235

Nissen, P. E., Akerman, C., Asplund, M., et al. 2007, A\&A, 469, 319

Nissen, P. E., \& Schuster, W. J. 2008, in The Galaxy Disk in Cosmological Context, ed. J. Andersen, J. Bland-Hawthorn, \& B. Nordstrom (Cambridge University Press), in press, IAU Symp., 254, 103

Péquignot, D. 2008, A\&A, 478, 371

Piskunov, N. E., Kupka, F., Ryabchikova, T. A., Weiss, W. W., \& Jeffery, C. S. 1995, A\&AS, 112, 525

Primas, F., Rebull, L. M., Duncan, D. K., et al. 2001, New Astron. Rev., 45, 541

Ramírez, I., Allende Prieto, C., \& Lambert, D. L. 2007, A\&A, 465, 271

Schuler, S. C., King, J. R., Hobbs, L. M., \& Pinsonneault, M. H. 2004, ApJ, 602, L117

Schuler, S. C., King, J. R., Hobbs, L. M., \& Pinsonneault, M. H. 2005, Nuclear Physics A, 758, 332

Schuler, S. C., King, J. R., Terndrup, D. M., et al. 2006, ApJ, 636, 432

Seaton, M. J. 1962, Proc. Phys. Soc., 79, 1105

Seaton, M. J. 1987, J. Phys. B, 20, 6363

Sedlmayr, E. 1974, A\&A, 31, 23

Shchukina, N. G. 1987, KFNT, 3, 36

Shchukina, N. G., \& Trujillo Bueno, J. 2001, ApJ, 550, 970

Shchukina, N. G., Trujillo Bueno, J., \& Asplund, M. 2005, ApJ, 618, 939

Sneden, C., Kraft, R. P., Prosser, C. F., \& Langer, G. E. 1991, AJ, 102, 2001

Spite, M., \& Spite, F. 1991, A\&A, 252, 689

Spite, M., Cayrel, R., Plez, B., et al. 2005, A\&A, 430, 655

Steenbock, W., \& Holweger, H. 1984, A\&A, 130, 319

Takeda, Y. 1992, PASJ, 44, 649

Takeda, Y. 1994, PASJ, 46, 53

Takeda, Y. 2003, A\&A, 402, 343

Takeda, Y., \& Honda, S. 2005, PASJ, 57, 65

van Regemorter, H. 1962, ApJ, 136, 906 